\title{
1 Binaural (pre)processing for contralateral sound field attenuation and improved 2 speech-in-noise recognition
}

3 Enrique A. Lopez-Poveda ${ }^{1,2,3,}{ }^{*}$, Almudena Eustaquio-Martín ${ }^{1,2}$, Fernando Martín San Victoriano ${ }^{1}$

$4{ }^{1}$ Laboratorio de Audición Computacional y Psicoacústica, Instituto de Neurociencias de Castilla

5 y León, University of Salamanca, 37007 Salamanca, Spain.

$6{ }^{2}$ Grupo de Audiología, Instituto de Investigación Biomédica de Salamanca, University of

7 Salamanca, 37007 Salamanca, Spain.

$8{ }^{3}$ Departamento de Cirugía, Facultad de Medicina, University of Salamanca, 37007 Salamanca, 9 Spain.

Running title: Binaural signal-to-noise ratio enhancement

12

* Author for correspondence:

Instituto de Neurociencias de Castilla y León

Universidad de Salamanca

Calle Pintor Fernando Gallego 1

Spain

Phone: (+34) 923294500 ext. \#1957

E-mail: ealopezpoveda@usal.es

Authors contributions: EALP designed the algorithm and the study. AEM performed the technical evaluations and wrote the software tools for the experimental evaluations. FMSV collected the experimental data with human participants. EALP wrote the manuscript. All authors analyzed the data and revised the manuscript. 


\section{ABSTRACT}

Understanding speech presented in competition with other sound sources can be challenging. Here, we reason that this task can be facilitated by improving the signal-to-noise ratio (SNR) in either of the two ears and that in free-field listening scenarios, this can be achieved by attenuating contralateral sounds. We present a binaural (pre)processing algorithm that improves the SNR in the ear ipsilateral to the target sound source by linear subtraction of the weighted contralateral stimulus. Although the weight is regarded as a free parameter, we justify setting it equal to the ratio of ipsilateral to contralateral head-related transfer functions averaged over an appropriate azimuth range. The algorithm is implemented in the frequency domain and evaluated technically and experimentally for normal-hearing listeners in simulated free-field conditions. Results show that (1) it can substantially improve the SNR (up to $20 \mathrm{~dB}$ ) and the short-term intelligibility metric in the ear ipsilateral to the target source, particularly for speech-like maskers; (2) it can improve speech reception thresholds for sentences in competition with speech-shaped noise by up to $8.5 \mathrm{~dB}$ in bilateral listening and $10.0 \mathrm{~dB}$ in unilateral listening; (3) it hardly affects sound-source localization; and (4) the improvements, and the algorithm's directivity pattern depend on the weights. The algorithm accounts qualitatively for binaural unmasking for speech in competition with multiple maskers and for multiple target-masker spatial arrangements, an unexpected property that can inspire binaural intelligibility models.

Keywords: noise reduction; binaural hearing; binaural unmasking; hearing aid; cochlear implant; hearing device 


\section{INTRODUCTION}

50 In daily listening scenarios, listeners often wish to attend to a sound source (the 'target') and ignore other, competing sound sources (the 'maskers'). For example, while dining in a restaurant, a listener may wish to understand what a friend is saying disregarding other talkers. It is not yet totally clear how listeners with normal hearing can solve this so-called 'cocktail party' problem (Cherry, 1953), but it is well documented that hearing-impaired people have more trouble at this task than do people with normal hearing. The signal-to-noise ratio (SNR) required to achieve the same amount of speech understanding (e.g., $50 \%$ sentence recognition) is up to $9 \mathrm{~dB}$ higher for hearing-impaired than for normal-hearing people (e.g., Festen and Plomp, 1990; Peters et al., 1998). For this reason, assistive listening devices, including hearing aids and cochlear implants, often incorporate pre-processing algorithms that improve the SNR before the stimulus is further processed and delivered to the user. Here, we present a binaural (pre)processing algorithm that enhances the SNR in the ear ipsilateral to the target source by attenuating the contralateral sound field.

There exist many different pre-processing approaches to improve the SNR (for a recent review, see Doclo et al., 2015). Most algorithms assume that some knowledge is available a priory to distinguish the target from the masker sources. Some algorithms are based on assumptions about the temporal or spectral characteristics of the target or the maskers and enhance the SNR by filtering out the maskers in the time-frequency domain (e.g., Boll, 1979). Other algorithms, known as beamformers, are based on assumptions about the spatial location of the target or the masker sources and enhance the SNR by spatial filtering out the masker sources (e.g., Cox et al., 1987). (Beamformers typically involve using two microphones in each device to attenuate masker sounds from the rear.) Other algorithms can blindly separate the multiple sound sources in the acoustic scene and improve the SNR by attenuating the masker sources. Once separated, the target source needs to be selected by the user or based on assumptions about its location or acoustic characteristics. Blind-source separation requires as many microphones as sound sources are to be separated, including the user's own voice (Hamacher et al., 2005; Doclo et al., 2015).

The approaches just described are monaural. For users of bilateral devices, monaural SNRenhancement algorithms are often applied separately in each device, an approach that can distort inter-aural time (ITD) and level differences (ILDs) and thus hinder the localization of sound sources (e.g., Cornelis et al., 2012; Doclo et al., 2015). The introduction of assistive listening devices that swap signals between the two ears has spawned the development of 
binaural pre-processing algorithms, i.e., systems that couple the functioning of two devices (Hamacher et al., 2005; Moore, 2007; Baumgärtel et al., 2015a, 2015b). One approach consists of detecting binaural cues and re-introducing them after the SNR is enhanced separately in each ear (see the review of Doclo et al., 2015). Another approach involves taking advantage of the greater number of microphones available in the two devices to perform blind separation of a greater number of sources (binaural source separation with only one microphone per ear does not improve intelligibility when there are three masker sources; Luts et al., 2010). Liu et al. (2001) proposed an algorithm that could localize multiple sound sources in a scene with only two microphones and improve the SNR by cancelling the unwanted sources. As with monaural systems, however, a limitation of binaural source separation or localization algorithms is that the target still needs to be selected by the user or differentiated from the maskers based on assumptions (Hamacher et al., 2005; Doclo et al., 2015).

Users of assistive listening devices often have reduced sensitivity to binaural cues or no access to them. Indeed, (1) some hearing-impaired users have reduced sensitivity to ITDs for narrowband stimuli (see Best and Swaminathan, 2019); (2) bilateral cochlear implants provide minimal access to ITDs (e.g., Grantham et al., 2008); and (3) the use of bilateral hearing aids and cochlear implants reduces or distorts ILDs when the two devices apply amplitude (or dynamic range) compression independently to each ear (e.g., Wiggins and Seeber, 2013; Lopez-Poveda et al., 2016a, 2019). Because binaural cues are important in 'cocktail party' listening situations (Bronkhorst and Plomp, 1989; but see Baltzell et al., 2020), some binaural pre-processing algorithms are designed to enhance naturally occurring binaural cues that may be reduced or absent for the listener. For example, some algorithms detect head-shadow ITDs, which are present at low frequencies $(<1500 \mathrm{~Hz})$ and convert them into ILDs. These algorithms can improve sound source localization for users of bilateral hearing aids (Moore et al., 2016) and cochlear implants (Brown, 2018). They can also help bilateral cochlear-implant users recognize speech in competition with a speech interferer (Brown, 2014). However, they have failed to improve speech-in-noise recognition for bilateral hearing-aid users (Moore et al., 2016). One important limitation of this approach is that ILD enhancement per se needs not improve the SNR. To improve the SNR, ILDs should be enhanced separately for each sound source. Kokkinakis and Pirhosseinloo (2017) proposed an algorithm that enhanced the SNR for a target in competition with a single masker by enhancing the ILD for the masker alone. This algorithm, however, requires knowing the specific location of the masker as well as the listener's head-related transfer function (HRTF) for the masker location, and this information needs not be available a priori. 
Here, we propose a binaural (pre)processing algorithm that improves the SNR in the ear

117 ipsilateral to the target by attenuating the contralateral sound field. The algorithm is inspired

118 by the binaural cochlear-implant sound processing strategy of Lopez-Poveda et al. (2016a,

$1192016 b, 2017,2018,2020)$. That strategy involved dynamic contralateral control of the

120 compression in each frequency channel so that the stronger spectro-temporal features in each

121 ear can suppress themselves in the contralateral ear. As a result, a sound source is enhanced in

122 the ipsilateral ear but attenuated in the contralateral ear. In cocktail-party scenarios with

123 multiple talkers, the target talker is unlikely centered (Grange and Culling, 2016) and listeners

124 can switch attention to the ear that has the better SNR for the momentary target (Bronkhorst

125 and Plomp, 1988; Brungart and lyer, 2012). Therefore, in cocktail party listening scenarios, the

126 strategy of Lopez-Poveda et al. (2016a) can improve speech intelligibility without making any

127 assumptions about the location of the target source ${ }^{1}$.

128 The algorithm proposed here was intended to produce a similar effect as the strategy of Lopez-

129 Poveda et al. by subtraction of the weighted contralateral stimulus. The weight is regarded as a

130 free parameter, but we propose setting it equal to the ratio of ipsilateral to contralateral

131 HRTFs for an anthropometric manikin averaged over an appropriate azimuth range (see

132 below). The algorithm is implemented in the frequency domain and evaluated technically and

133 experimentally for listeners with normal hearing.

\section{THE ALGORITHM}

In natural listening situations, our brain has access only to the pressure waveforms sensed by the left and right eardrums. In a free-field acoustic scenario where a target sound source, $S(t)$, is presented in competition with $n$ masker sources $\left[M_{i}(t)\right.$, for $\left.i=1 \ldots n\right]$, the waveforms at the left and right ear, $L(t)$ and $R(t)$, respectively, can be expressed as a linear summation of the pressure waveforms generated by all sources, each convolved with the corresponding headrelated impulse response (HRIR), as follows:

$$
\begin{gathered}
L(t)=S(t) * H_{S L}(t)+\sum_{i=1}^{n}\left[M_{i}(t) * H_{M_{i} L}(t)\right] \\
R(t)=S(t) * H_{S R}(t)+\sum_{i=1}^{n}\left[M_{i}(t) * H_{M_{i} R}(t)\right],
\end{gathered}
$$

\footnotetext{
${ }^{1}$ For sound sources in the left hemifield, the algorithm improves the SNR in the left ear by attenuating sound sources in the right hemifield. For sources in the right hemifield, the algorithm improves the SNR in the right by attenuating sound sources in the left hemifield. The listener can then attend to the rightear or the left-ear stimulus, which ever has the higher SNR for the target, depending on whether the target is in the right or the left hemifield.
} 
where $t$ denotes time; $H_{S L}(t)$ and $H_{S R}(t)$ denote the HRIRs for the signal source for the left and right ears, respectively; $H_{M_{i} L}(t)$ and $H_{M_{i} R}(t)$ denote the HRIRs for the $i$-th masker, $M_{i}(t)$, for the left and right ears, respectively; and asterisks $\left({ }^{*}\right)$ denote the convolution operator.

Thanks to the properties of the Fourier transform, Eq. (1) may be expressed in the frequency domain as follows:

$$
\begin{array}{r}
L(f)=S(f) \cdot H_{S L}(f)+\sum_{i=1}^{n}\left[M_{i}(f) \cdot H_{M_{i} L}(f)\right] \\
R(f)=S(f) \cdot H_{S R}(f)+\sum_{i=1}^{n}\left[M_{i}(f) \cdot H_{M_{i} R}(f)\right]
\end{array}
$$

where $f$ denotes frequency, each time waveform in Eq. (1) has been replaced by its corresponding complex frequency spectrum in Eq. (2), the HRIRs have been replaced with the corresponding frequency-domain complex HRTFs, and the convolution operator has been replaced by a product.

Let $L^{\prime}(f)$ and $R^{\prime}(f)$ denote the frequency spectra of the processed stimuli. The proposed approach consists of subtracting the weighted, unprocessed contralateral stimulus from the stimulus in each ear, as follows:

$$
\begin{aligned}
& L^{\prime}(f)=L(f)-\beta(f) \cdot R(f) \\
& R^{\prime}(f)=R(f)-\alpha(f) \cdot L(f)
\end{aligned}
$$

where $\alpha(f)$ and $\beta(f)$ are the subtraction weights.

The subtraction weights are regarded as free parameters and complex numbers (i.e., with magnitude and phase). Therefore, they may be set to any appropriate values that improve the SNR. In the Appendix, it is mathematically shown that the values of $\alpha(f)$ and $\beta(f)$ that would optimally cancel all maskers in the left and right ears would be as follows:

$$
\begin{aligned}
& \beta(f)=\frac{\sum_{i=1}^{n} M_{i}(f) H_{M_{i} L}(f)}{\sum_{i=1}^{n} M_{i}(f) H_{M_{i} R}(f)} \\
& \alpha(f)=\frac{\sum_{i=1}^{n} M_{i}(f) H_{M_{i} R}(f)}{\sum_{i=1}^{n} M_{i}(f) H_{M_{i} L}(f)}
\end{aligned}
$$

Equations (4a) and (4b) show that aiming for a perfect cancellation of the maskers at the two ears would be impractical because it would require knowing the spectrum of every masker, $M_{i}$ $(f)$, as well as the HRTFs for each individual listener and for each masker location for the left and the right ear $\left[H_{M_{i} L}(f)\right.$ and $\left.H_{M_{i} R}(f)\right]$. This information needs not be available a priori. Furthermore, in many listening situations, the identity and location of the signal and masker 
171

172

sources, thus the spectra of the maskers, change from moment to moment. For instance, in 'cocktail party' listening situations, the target and masker speakers often change during the conversation, and so do their locations relative to the listener as the listener moves the head and/or the target and masker talkers move around the listener. This makes it impractical, if not impossible, to calculate $\alpha(f)$ and $\beta(f)$ for optimal cancellation of the masker(s) in an arbitrary listening situation.

Because $\alpha(f)$ and $\beta(f)$ are free parameters, however, they can be set to any appropriate value for a specific purpose. In what follows, we set:

$$
\begin{gathered}
\beta_{\text {diff }}(f)=\frac{\sum_{\theta=0}^{180} H_{\theta L}(f)}{\sum_{\theta=0}^{180} H_{\theta R}(f)}, \\
\alpha_{\text {diff }}(f)=\frac{\sum_{\theta=180}^{360} H_{\theta R}(f)}{\sum_{\theta=180}^{360} H_{\theta L}(f)},
\end{gathered}
$$

where $\theta$ denotes azimuthal angle, going from $0^{\circ}$ for a source right in front to $360^{\circ}$ clockwise, and $H_{\theta L}(f)$ and $H_{\theta R}(f)$ denote the complex HRTFs for an average listener. The appropriateness of this choice is discussed later. For convenience, in what follows, we will use the notation $\beta_{\text {diff }}$ and $\alpha_{\text {diff } f}$ rather than $\beta_{\text {diff }}(f)$ and $\alpha_{\text {diff }}(f)$.

Note that for a symmetrical head:

$$
\sum_{\theta=0}^{180} H_{\theta L}(f)=\sum_{\theta=180}^{360} H_{\theta R}(f) \quad \text { and } \quad \sum_{\theta=0}^{180} H_{\theta R}(f)=\sum_{\theta=180}^{360} H_{\theta L}(f)
$$

Hence:

$$
\beta_{\text {diff }}=\alpha_{\text {diff }}
$$

The rest of this paper is devoted to showing that this algorithm (Eq. 3) with $\alpha_{\text {diff }}$ and $\beta_{\text {diff }}$ can improve the SNR in the ear contralateral to the masker source and thus intelligibility of speech in noise without significantly altering the lateralization of sound sources. Before doing so, however, several comments are in order.

1. We propose that $\alpha_{\text {diff }}$ and $\beta_{\text {diff }}$ be calculated using average, rather than individual, HRTFs. In other words, our proposed approach does not require using individualized HRTFs. In what follows $\alpha_{\text {diff }}$ and $\beta_{\text {diff }}$ were calculated for a KEMAR, an anthropometric acoustic manikin with average body dimensions (Burkhard and Sachs, 1975). Their magnitude and phase are shown in Fig. 1. The effect of calculating $\alpha_{\text {diff }}$ and $\beta_{\text {diff }}$ using individualized and non-individualized HRTFs is analyzed and discussed below. 
2. The proposed algorithm (Eq. 3) is linear. Therefore, the waveform that results from applying the algorithm to the signal+masker stimulus is equal to adding the waveforms that result from applying the algorithm to the signal and the masker separately, i.e.:

$$
F E(\text { signal }+ \text { maskers })=F E(\text { signal })+F E(\text { maskers }),
$$

where $F E(x)$ denotes the front-end algorithm applied to signal $x$. The linearity property is important because it implies that the algorithm works equally at all signal and masker levels, i.e., across SNRs.

3. $\beta_{\text {diff } f}$ is the theoretical optimal $\beta(f)$ to provide an infinite SNR (i.e., to perfectly cancel all maskers) in the left ear in a hypothetical listening scenario with infinite, identical $\left[M_{i}(f)\right.$ $=M(f)$ for all $i$ ] point-source maskers located at azimuthal angles from $0^{\circ}$ to $180^{\circ}$. In other words, $\beta_{\text {diff } f}$ is the optimal weight for the algorithm to provide an infinite SNR in the left ear for a 'diffuse-field' masker in the right hemifield. Similarly, $\alpha_{\text {diff }}$ would be the optimal weight to deliver an infinite SNR in the right ear for a 'diffuse-field' masker in the left hemifield. With $\alpha_{d i f f}$ and $\beta_{\text {diff }}$, the algorithm does not provide perfect cancellation in any other condition, but still improves the SNR in many listening situations, as shown below.

4. The algorithm results in the directivity patterns shown in Fig. 2. (Note that the pattern is shown only for the left ear because the pattern for the right ear would be symmetrical about the midline.) The algorithm attenuates contralateral sound sources at low frequencies $(0-1500 \mathrm{~Hz})$, particularly at azimuths about $45^{\circ}$ and $135^{\circ}$, and it slightly enhances contralateral high frequencies $(>1500 \mathrm{~Hz})$ at the same azimuths (Fig. 2B). This can enhance the SNR in the ear contralateral to the masker, for masker frequencies below about $1500 \mathrm{~Hz}$.

5. The directivity pattern is similar for white noise (Fig. 2A, B) and speech-shaped noise (Fig. 2C, D). However, the overall SNR enhancement is expected to be greater for speechshaped noise than for white noise because speech-shaped noise has more low-frequency content and less high-frequency content than white noise (compare Fig. 3 and Fig. 4). The SNR enhancement effect of the algorithm for different masker types is analyzed and discussed below. 
6. The algorithm attenuates contralateral low-frequency sounds with minimal changes in the level of ipsilateral sounds (Fig. 2). This distorts the head-shadow ILDs. This distortion and its effect on sound localization is analyzed and discussed below.

\section{MATERIALS AND METHODS}

\section{Evaluations}

The proposed algorithm (Eq. 3) with $\alpha_{\text {diff }}$ and $\beta_{\text {diff }}$ (Eq. 5) was evaluated technically and experimentally. The technical evaluation involved comparing the short-term objective intelligibility (STOI) (Taal et al., 2011) as well as the SNR for processed versus unprocessed stimuli. The experimental evaluation involved comparing the SNR at 50\% speech recognition (termed speech reception threshold or SRT in noise) as well as sound-source lateralization performance in quiet for listeners with normal hearing for processed versus unprocessed stimuli. $\alpha_{\text {diff }}$ and $\beta_{\text {diff }}$ (shown in Fig. 1) were always calculated using the HRTF database for a KEMAR (Gardner and Martin, 1995).

All evaluations were performed in a simulated free-field. All sound sources were at eye level ( $0^{\circ}$ elevation). To achieve the desired target-masker spatial configurations, monophonic sound recordings were filtered through diffuse-field HRTFs. Unless otherwise stated, the same KEMAR HRTF database that was used to calculate $\alpha_{\text {diff } f}$ and $\beta_{\text {diff } f}$ was also used to simulate free-field listening. This would be equivalent to calculating $\alpha_{\text {diff }}$ and $\beta_{\text {diff } f}$ using individualized HRTFs. For a subset of conditions (see below), however, we also assessed the benefits provided by algorithm when using non-individualized $\alpha_{\text {diff }}$ and $\beta_{\text {diff }}$. In this case, the spatial location of the sound sources was simulated by filtering stimuli through a different set of HRTFs (from IRCAM subject $1017^{2}$ ).

Experimental tests were approved by the Ethics Committee of the University of Salamanca. Participants were volunteers and not paid for their services. All of them signed an informed consent to participate in the study.

\footnotetext{
${ }^{2}$ http://recherche.ircam.fr/equipes/salles/listen/download.html
} 


\section{Implementation}

258 The algorithm was implemented in the frequency domain. Monophonic time-domain input waveforms for the target and masker(s) were first linearly scaled to the desired level. Here, levels are expressed in decibels relative to a maximum amplitude of unity and denoted as $\mathrm{dB}$ FS (full scale). The scaled, monophonic waveforms were convolved through appropriate HRIRs to obtain the unprocessed waveforms at the left and right ears, $L(t)$ and $R(t)$, for the desired (simulated) free-field target-masker spatial configuration. The stimulus waveforms were then subject to a frame-based processing to obtain their corresponding complex frequency spectra. To do it, the input waveform in each ear was divided into time-overlapping frames of 128 samples (the frame overlapping was 64 samples). Each signal frame was multiplied with a Hanning window and the result was padded with zeros to obtain a frame of 256 samples (64 samples of zeros were added before and after the signal frame). A fast Fourier transform (FFT) was applied to the windowed, zero-padded frame to obtain the complex frequency spectrum of the frame. The processing algorithm (Eq. 3) was applied to the resulting complex spectrum for each frame, and an inverse FFT was applied to obtain the processed waveforms, $L^{\prime}(t)$ and $272 R^{\prime}(t)$.

\section{Signal-to-noise ratio}

For unprocessed stimuli, the SNR (in decibels) was calculated separately in each ear as follows:

$$
S N R_{\text {in }}=20 \log _{10}\left[\frac{R M S(\text { target })}{R M S(\text { masker })}\right]
$$

Where RMS(target) and RMS(masker) denote the root-mean-square (RMS) amplitude of the waveform in the ear in question for the HRTF-filtered target alone and the HRTF-filtered masker(s) alone, respectively.

Similarly, the SNR for processed stimuli was calculated in each ear as follows:

$$
S N R_{\text {out }}=20 \log _{10}\left[\frac{R M S(F E(\text { target }))}{R M S(F E(\text { masker }))}\right]
$$

Where FE(target) and FE(masker) denote the waveforms at the output of the algorithm for the HRTF-filtered target alone and the HRTF-filtered masker(s) alone, respectively.

The SNR improvement $(\mathrm{dB})$ provided by the algorithm was calculated as:

$$
\Delta S N R=S N R_{\text {out }}-S N R_{\text {in }}
$$


Positive $\triangle S N R$ values indicate that the algorithm improved the SNR (i.e., that the SNR was greater for processed than unprocessed stimuli) while negative values indicate the algorithm decreased the SNR.

Note that $S N R_{\text {out }}$ may be calculated using Eq. (10) because the algorithm is linear (Eq. 8). Also note that in the above calculations, the RMS amplitudes were calculated based on the input and output time waveforms to and from the processor, even though the algorithm was implemented in the frequency domain.

\section{Short-term objective intelligibility}

The STOI is an objective measure of the amount of speech information available when the speech is degraded by a masker and/or processed by a sound processor (Taal et al., 2011). It is the average linear correlation over time and frequency between the unprocessed speech in quiet and the processed speech in noise. It is a scalar value between 0 and 1 that is expected to have a monotonic relation with the percentage of correctly understood speech tokens averaged across a group of listeners. Therefore, higher STOI values indicate higher SNR and better intelligibility in noise.

Here, the proposed algorithm was evaluated by calculating how much it improved the STOI. The STOI improvement, $\Delta$ STOI, was calculated as follows:

$$
\Delta S T O I=S T O I[\text { target }, F E(\text { target }+ \text { masker })]-S T O I[\text { target },(\text { target }+ \text { masker })]
$$

Where the notation $\operatorname{STOI}\left(x_{\text {ref }}, x\right)$ indicates that STOI was calculated for stimulus $x$ having stimulus $x_{\text {ref }}$ as the reference. In other words, positive $\triangle$ STOI values indicate that intelligibility, as predicted by STOI, would be greater for processed, FE(target+masker), than for unprocessed (target+masker) stimulus. STOI was calculated separately for the left and right ears having HRTF-filtered stimuli as the reference.

Unless otherwise stated, in calculating STOI, the target consisted of 10 concatenated sentences (with pre- and post-silence periods removed) from practice list \#1 of the Castilian-Spanish hearing-in-noise test (HINT) (Huarte et al., 2008). STOI was calculated for three types of maskers: Gaussian white noise (WN), Gaussian noise filtered to have the long-term spectrum of speech (referred to as speech-shaped noise or SSN), and an international female fluctuating masker (IFFM) (Holube et al., 2010). The levels of the target and the masker were set equal to $-20 \mathrm{~dB} F S$ (i.e., the SNR was $0 \mathrm{~dB}$ ). The masker waveform was identical for processed and unprocessed stimuli to allow a fair comparison. 


\section{Speech recognition tests}

317 For listeners with normal hearing (NH), SRTs for sentences in noise were compared for processed and unprocessed stimuli. Speech reception thresholds were measured in bilateral and unilateral listening modes. Unilateral listening tests involved processing stimuli through the algorithm but stimulating only the ear ipsilateral to the target source (i.e., the left ear in this case, see below). Unilateral listening tests were aimed at testing to what extent $\triangle S N R$ (which is calculated for each ear) is a reasonable predictor of the actual SRT improvement.

Procedures. SRTs were measured using an adaptive procedure. The target was a sentence from the Spanish matrix sentence test (Hochmuth et al., 2012) and the masker was steadystate noise with the average long-term spectrum of the matrix sentences. During an SRT measurement, the speech level ${ }^{3}$ was fixed at $50 \mathrm{~dB}$ SPL and the noise level varied adaptively using a one-down, one-up rule. The SRT was thus defined as the SNR at which listeners recognized $50 \%$ of full sentences (Levitt, 1971). Thirty sentences were presented to measure an SRT. The first 10 sentences were always the same (randomly presented) and were included to give listeners an opportunity to become familiar with the task. The initial SNR was $20 \mathrm{~dB}$ and it changed in 4-dB steps between sentences 1 and 14 and 2-dB steps between sentences 14 and 30. The SRT was calculated as the mean of the final 17 SNRs (the SNR for the 31st sentence was calculated and used in the SRT estimate but not actually presented). Each SRT estimate was measured three times and the mean was taken as the final SRT.

Conditions. SRTs were measured for three spatial configurations of target and masker sources: $\mathrm{S}_{0} \mathrm{~N}_{0}$ with the speech and noise sources collocated in front of the listener at $0^{\circ}$ azimuth; $\mathrm{S}_{270} \mathrm{~N}_{45}$ with the speech and noise sources at $270^{\circ}$ and $45^{\circ}$ azimuth, respectively; and $\mathrm{S}_{270} \mathrm{~N}_{90}$ with the speech and noise sources at $270^{\circ}$ and $90^{\circ}$ azimuth, respectively. These spatial configurations were selected as representative of conditions where the STOI and SNR evaluations predicted the benefit from the algorithm to be largest $\left(S_{270} N_{45}\right)$, intermediate $\left(S_{270} N_{90}\right)$, or none $\left(S_{0} N_{0}\right)$ (see below).

In total, 36 SRTs were measured per participant: 2 listening modes (bilateral and unilateral) $\times 2$ processing conditions (with and without processing) $\times 3$ spatial configurations $\left(\mathrm{S}_{0} \mathrm{~N}_{0}, \mathrm{~S}_{270} \mathrm{~N}_{45}\right.$ and $\left.\mathrm{S}_{270} \mathrm{~N}_{90}\right) \times 3$ SRT estimates per condition. Measurements were organized in three blocks of

\footnotetext{
${ }^{3}$ The speech level was lower than used in most conventional tests (50 rather than $65 \mathrm{~dB}$ SPL) because the technical evaluation of the algorithm predicted that SRTs in noise would improve up to $14 \mathrm{~dB}$ SNR in some conditions, and hence the noise level at the SRT would have been high ( $90 \mathrm{~dB}$ SPL) and uncomfortable if the speech level had been $65 \mathrm{~dB}$ SPL.
} 
12 SRT measurements each, one block per SRT estimate. Within each block, bilateral and unilateral listening modes were alternated and for each listening mode conditions were administered in random order. Participants did not know which condition they were being tested on.

Participants. Five NH adults (three women) participated in the tests. They were all native Spanish speakers. Their age range was 25 to 28 years, and their absolute hearing thresholds were less than $20 \mathrm{~dB} \mathrm{HL}$ at the audiometric frequencies from 125 to $8000 \mathrm{~Hz}$.

\section{Sound localization tests}

For NH listeners, sound source localization in a virtual horizontal plane was assessed for processed and unprocessed stimuli.

Stimuli. For each processing condition, localization was assessed for lowband (125-1000 Hz) and broadband (125-6000 Hz) noise bursts (four test conditions in total). Gaussian noise bursts were digitally generated and bandpass filtered (first-order Butterworth filter) to achieve the desired bandwidth. Stimuli had a duration of $200 \mathrm{~ms}$ (including 50-ms raised-cosine onset and offset ramps) and their level was fixed at $65 \mathrm{~dB}$ SPL. No level roving was applied to minimize the use of monaural level cues.

Procedure. The procedure was virtually identical to that of Lopez-Poveda et al. (2019). For each test condition, participants were presented with eight noise tokens for each one of the 13 azimuthal angles in the frontal horizontal plane from $270^{\circ}$ to $90^{\circ}$, spaced every $15^{\circ}$ (104 noise tokens in total). The 104 noise tokens were presented in random order. During the presentation of the stimuli, participants sat in front of a computer screen that displayed a top view of a human head with an array of speakers in front of the head. For each stimulus presentation, the subject was instructed to judge the azimuthal position of the sound source by clicking on the corresponding speaker on the computer screen. The click of a response triggered the processing of a freshly generated noise stimulus through the corresponding condition, and the presentation of the stimulus to the participant. The response screen displayed an array of 37 speakers spaced every $5^{\circ}$ over an azimuth range from $270^{\circ}$ to $90^{\circ}$, even though stimuli were presented every $15^{\circ}$. Feedback was not given to participants on the correctness of their responses.

Before data collection began, participants were encouraged to train themselves on the task by clicking on any of 37 speakers evenly spaced every $5^{\circ}$ over an azimuth range from $270^{\circ}$ to $90^{\circ}$ and listening to the corresponding stimulus (that is, during training, participants could hear 
377 stimuli at all those azimuthal locations while for testing, stimuli were presented at a subset of

378 locations). Training was provided independently for each test condition and for as long as each participant deemed necessary. The four test conditions were administered in random order and participants did not know which condition they were training on or being tested with.

Participants. Five NH adults (three women) participated in the experiment. Their age range was 26 to 31 years, and their absolute hearing thresholds were less than $20 \mathrm{~dB} \mathrm{HL}$ at the audiometric frequencies from 125 to $8000 \mathrm{~Hz}$. Three of these participants also participated in the speech-in-noise recognition test.

\section{Equipment}

The MATLAB software environment (R2017b, The Mathworks, Inc.) was used to perform all signal processing and implement all test procedures. All technical and experimental evaluations were conducted with digital stimuli sampled at a rate of $44100 \mathrm{~Hz}$ and with 24-bit resolution. For the experimental tests, stimuli were controlled using custom-made software and played via an RME Fireface UCX soundcard and presented to the listeners via ER-2 insert earphones (Etymotic Research Inc., Elk Grove Village, Illinois). Sound pressure levels (SPLs) were calibrated by placing the earphones in a Zwislocki DB-100 coupler connected to a sound level meter (Brüel\&Kjaer, mod. 2238). Calibration was performed at $1 \mathrm{kHz}$ and the obtained sensitivity was used at all other frequencies.

\section{RESULTS}

\section{SNR and STOI improvement for a single SSN source}

397 Figure 5 shows the SNR improvement (Eq. 11) provided by the algorithm in each ear, for a 398 target speech source in competition with a single source of SSN, and for multiple azimuthal 399 locations of the target and masker sources in the horizontal plane ( $0^{\circ}$ elevation). The level of 400 the target and the masker before HRTF filtering was $-20 \mathrm{~dB}$ FS (i.e., $0 \mathrm{~dB}$ SNR). The pattern of results in each panel (i.e., for each ear) suggests four quadrants, bounded by the dotted lines, and identified with numbers 1 to 4 . Note that the quadrants are symmetrical for the left and right ears and so is the numbering. Quadrant 1 is for an ipsilateral target with a contralateral masker. In this case, the processor improves the SNR for most spatial configurations. The improvement can be as large as $14 \mathrm{~dB}$ and is caused by the attenuation of 
407 masker. In this case, the processor hardly alters the SNR. Quadrant 3 is for a contralateral

408 target with a contralateral masker. In this case, the processor can increase or decrease the 409 SNR, depending on the actual azimuthal locations of the target and the masker. Quadrant 4 is 410 for an ipsilateral masker with a contralateral target. In this case, the processor decreases the 411 SNR overall because it attenuates the contralateral target (Fig. 2). Importantly, overall, the processor tends to improve the SNR in the ear ipsilateral to the target.

It can be mathematically demonstrated (not shown) that the algorithm does not alter the SNR in two special cases: (1) for collocated target and masker sources (i.e., along the dashed-dotted diagonals); and (2) for ipsilateral target and masker sources that are symmetrically placed with respect to the left-right axis (i.e., for a target at $45^{\circ}$ and a masker at $135^{\circ}$ ).

Figure 6 shows the STOI improvement (Eq. 12) provided by the algorithm with $\beta_{\text {diff }}$ and $\alpha_{\text {diff }}$ for the same spatial locations of the target and masker sources as shown in Fig. 5. The pattern of results matches the pattern of SNR improvement illustrated in Fig. $\mathbf{5}$.

\section{SNR and STOI improvement for multiple maskers and masker types}

In the preceding section, it has been shown that the algorithm can improve the SNR and STOI in the ear ipsilateral to the target for simulated free-field listening conditions where the target speech source is in competition with a single source of SSN. Figure 7 illustrates STOI and SNR improvements for listening conditions involving one or more simultaneous maskers and for three different masker types: IFFM, SSN, and WN. The improvement is shown only for the ear ipsilateral to the target, if any. For conditions involving multiple masker sources, the waveforms for all maskers were different. The only exception is indicated with the suffix "eq." (for equal), where all masker sources had identical waveforms. For completeness, Fig. 7 shows results for $\alpha_{\text {diff }}$ and $\beta_{\text {diff }}$ calculated using individualized and non-individualized HRTFs (see Materials and Methods). In these calculations, the target and masker levels were set to -40 and $-30 \mathrm{~dB} F \mathrm{~S}$, respectively (i.e., the SNR was $-10 \mathrm{~dB}$ ).

The results in Fig. 7 are grouped, from top to bottom, in 10 blocks of three masker types each. 
azimuths of $0^{\circ}, 345^{\circ}, 330^{\circ}$, and $270^{\circ}$ ) in competition with three simultaneous maskers at various locations, as indicated in the figure. Blocks \#8 and \#9 involve a target at $0^{\circ}$ azimuth in competition with 37 identical (eq.) and different maskers, respectively, spanning the azimuth range from $0^{\circ}$ to $180^{\circ}$ (in $5^{\circ}$ steps). Lastly, block \#10 involves a target at $0^{\circ}$ azimuth in competition with 72 different maskers spanning the azimuth range from $0^{\circ}$ to $360^{\circ}$ (in $5^{\circ}$ steps).

Figure 7 shows the following. (1) For most conditions, $\triangle S N R \geq 0 \mathrm{~dB}$ and $\triangle S T O I \geq 0$ indicating that the algorithm improves the SNR and STOI. The exceptions were condition \#1, with collocated target and masker sources, and condition \#10, with maskers all around from $0^{\circ}$ to $355^{\circ}$. (2) For every condition, the improvement is comparable for IFFM and SSN, and smaller for WN. (3) Improvements are overall larger when $\alpha_{\text {diff }}$ and $\beta_{\text {diff }}$ are calculated using individualized than non-individualized HRTFs. However, important improvements are obtained even with nonindividualized HRTFs. (4) The largest improvements occur for condition \#8, with individualized HRTFs in a listening scenario with identical (equal) maskers on the contralateral hemifield, i.e., azimuths from $0^{\circ}$ to $180^{\circ}$. This is not surprising because $\alpha_{\text {diff } f}$ and $\beta_{\text {diff } f}$ were designed (Eq. 5) to produce perfect masker cancellation precisely in this hypothetical listening scenario.

\section{Speech recognition in noise}

While the SNR and STOI evaluations described earlier suggest that the proposed algorithm can improve the intelligibility of speech in competition with a single noise source, neither of them allows a direct assessment of the benefit provided by the algorithm in this task. For this reason, the algorithm was also evaluated experimentally by comparing SRTs for sentences in competition with a single SSN source. The evaluation was restricted to three spatial configurations of the target and masker sources $\left(\mathrm{S}_{0} \mathrm{~N}_{0}, \mathrm{~S}_{270} \mathrm{~N}_{45}\right.$, and $\left.\mathrm{S}_{270} \mathrm{~N}_{90}\right)$, where the technical evaluations just described predicted the benefit to be none, large, and medium, respectively (see blocks \#1 to \#3 in Fig. 7).

Figure 8A allows a comparison of individual and group mean SRTs in bilateral listening for processed and unprocessed stimuli, for the three spatial configurations. Lower SRT values indicate better performance. Figure $\mathbf{8 B}$ shows the SRT improvement provided by the algorithm. A two-way RMANOVA was conducted to test for the effect of processing (processed, unprocessed) and spatial configuration $\left(\mathrm{S}_{0} \mathrm{~N}_{0}, \mathrm{~S}_{270} \mathrm{~N}_{45}, \mathrm{~S}_{270} \mathrm{~N}_{90}\right)$ on the SRT. The test revealed a significant main effect of processing $[F(1,4)=218.26, p<0.001]$. Mean SRTs (across locations and participants) were significantly better for processed than for unprocessed stimuli 
( $-16.8 \mathrm{vs}-13.6 \mathrm{~dB}$ SNR, respectively). It also revealed a significant main effect of spatial configuration $[F(2,8)=1090.68, p<0.001]$. Post-hoc pairwise tests with Bonferroni correction showed that SRTs were significantly worse (higher) in the collocated condition than in any of the spatially separated conditions $\left[p\left(S_{0} N_{0}\right.\right.$ vs $\left.S_{270} N_{45}\right)<0.001 ; p\left(S_{0} N_{0}\right.$ vs $\left.S_{270} N_{90}\right)<0.001$ ], and they were significantly better in the $S_{270} \mathrm{~N}_{45}$ than in the $S_{270} \mathrm{~N}_{90}$ condition $(p<0.001)$. The interaction between processor and spatial configuration was also significant $[F(2,8)=138.31, p<0.001]$. Post-hoc pairwise comparisons with Bonferroni correction showed that the algorithm improved SRTs significantly only in the $S_{270} \mathrm{~N}_{45}$ spatial configuration $(p<0.001)$, but not in the $\mathrm{S}_{0} \mathrm{~N}_{0}(\mathrm{p}=0.290)$ or the $\mathrm{S}_{270} \mathrm{~N}_{90}(\mathrm{p}=0.063)$ configurations.

Figure 8C-D show the effect of the algorithm on SRTs when listening with the left ear alone, i.e., the processing was binaural, but stimuli were presented to the ear ipsilateral to the target source. A two-way RMANOVA revealed a significant main effect of processing $[F(1,4)=577.4$, $p<0.001]$. Mean SRTs (across locations and participants) were significantly better for processed than for unprocessed stimuli ( $-16.0 \mathrm{vs}-11.8 \mathrm{~dB}$ SNR, respectively). It also revealed a significant main effect of spatial configuration $[F(2,8)=801.63, p<0.001]$. Post-hoc pairwise tests with Bonferroni correction showed that SRTs were significantly worse (higher) in the collocated condition than in any of the spatially separated conditions $\left[p\left(S_{0} N_{0} v s S_{270} N_{45}\right)<0.001\right.$; $\mathrm{p}\left(\mathrm{S}_{0} \mathrm{~N}_{0}\right.$ vs $\left.\left.\mathrm{S}_{270} \mathrm{~N}_{90}\right)<0.001\right]$, and they were significantly better in the $\mathrm{S}_{270} \mathrm{~N}_{45}$ than in the $\mathrm{S}_{270} \mathrm{~N}_{90}$ condition $(p<0.001)$. The interaction between processor and spatial configuration was also significant $[F(2,8)=158.99, p<0.001]$. Post-hoc pairwise comparisons with Bonferroni correction showed that the algorithm improved SRTs significantly in the $S_{270} \mathrm{~N}_{45}(p<0.001)$ and $\mathrm{S}_{270} \mathrm{~N}_{90}$ $(p=0.002)$ but not in the $S_{0} N_{0}(p=0.886)$ conditions.

\section{Sound source localization}

As suggested by the directivity patterns of Fig. 2, the algorithm with $\beta_{\text {diff } f}$ and $\alpha_{\text {diff } f}$ can attenuate contralateral low frequencies $(<1500 \mathrm{~Hz})$ and slightly enhance high frequencies $(>1500 \mathrm{~Hz})$, distorting head-shadow ILDs. Figure 9 illustrates this effect more clearly by illustrating the level at each ear and the corresponding ILD as a function of sound source azimuth for white $(0-22050 \mathrm{~Hz})$, broadband $(125-6000 \mathrm{~Hz})$, and lowband $(125-1000 \mathrm{~Hz})$ noises of $-20 \mathrm{~dB}$ FS. Note that the algorithm distorts the stimulus level, particularly in the contralateral ear, and that the distortion is comparatively greater for lowband noise than for broadband or white noises. 
To assess the potential impact of these distortions, sound source location was assessed for unprocessed and processed lowband (LBN) and broadband (BBN) noises. Performance was quantified using the root mean square angle error between presentation and response angles.

Figure 10 shows a comparison of individual and group mean angle error for the four to test for the effects of the processing (processed and unprocessed) and noise type (LBN and $B B N$ ) on localization. The RMANOVA revealed that neither the processing $[F(1,4)=0.50, p=0.52]$ nor the noise bandwidth $[F(1,4)=0.37, p=0.58]$ had a statistically significant main effect on the angle error. The RMANOVA, however, revealed a statistically significant interaction between processing and noise type on angle error $[F(1,4)=38.8, p=0.003]$. Post-hoc pairwise comparisons with Bonferroni correction, however, revealed that the algorithm did not have a significant effect on the angle error for $\operatorname{LBN}(p=0.720)$ or $B B N(p=0.100)$.

\section{DISCUSSION}

517 We have proposed a pre-processing algorithm (Eq. 3) to attenuate the contralateral sound 518 field in each ear by weighted subtraction of the contralateral stimulus. The weights for the left and right ear $[\beta(f)$ and $\alpha(f)]$ are regarded as free parameters. Here, we have set them equal to $\beta_{\text {diff }}$ and $\alpha_{\text {diff }}$, respectively, and calculated them with Eq. (5) using KEMAR HRTFs. With these weights, the algorithm can improve the SNR (Figs. 5 and 7) and the intelligibility of speech in competition with one (Figs. 6 and 8) or more interfering sound sources (Fig. 7), with minimal impact on sound source localization (Fig. 10).

525 The specific weights employed here, $\beta_{\text {diff }}$ and $\alpha_{d i f f}$, were chosen ad hoc with the aim to 526 cancel a 'diffuse' masker in the contralateral hemifield, spanning the azimuthal range from $0^{\circ}$

527 to $180^{\circ}$ (see Eq. 5). These weights, however, need not be optimal or suitable for all 528 applications. Indeed, the algorithm produces different SNR improvements depending on the 529 weights. Figure 11 shows that for a target at $0^{\circ}$ azimuth in competition with a single SSN 530 source at azimuths from $0^{\circ}$ to $360^{\circ}$, the SNR improvement in the left ear would be different depending on the value of $\beta(f)$. In these examples, the values of $\beta(f)$ were calculated using Eq. (5b) but averaging HRTFs over different azimuth ranges, as indicated by the inset (note that 
$533 \beta_{\text {diff }}$ involved averaging KEMAR HRTFs over an azimuth range from $0^{\circ}$ to $\left.180^{\circ}\right)$. The figure

534 shows that while $\beta_{\text {diff }}$ causes SNR improvements to peak at masker azimuths of $45^{\circ}$ and $135^{\circ}$,

535 other $\beta(f)$ values can produce larger improvements and at different masker azimuths.

536 On the other hand, $\alpha_{\text {diff } f}$ and $\beta_{\text {diff } f}$ were appropriate to produce the sought effect of

537 attenuating contralateral maskers but only when maskers were low frequencies. With $\alpha_{\text {diff }}$

538 and $\beta_{\text {diff }}$, however, the algorithm enhanced contralateral high-frequency maskers slightly (Fig.

539 2). As a result, $\alpha_{\text {diff }}$ and $\beta_{\text {diff }}$ produce larger SNR improvements for maskers with relatively

540 weaker than stronger high-frequency content ( $>1500 \mathrm{~Hz})$. Because white noise has more high-

541 frequency content than IFFM or SSN, this explains why $\triangle$ SNR and $\triangle$ STOI tended to be smaller

542 for white noise than for IFFM or SSN (see Fig. 7 and also compare Fig. 3 vs. Fig. 4). Setting $\alpha_{\text {diff }}$

543 and $\beta_{\text {diff }}$ equal to zero for frequencies above $1500 \mathrm{~Hz}$ would prevent the amplification of

544 contralateral high-frequencies, which would extend the benefit from the algorithm to maskers

545 with strong high-frequency content, including white noise.

\section{$547 \quad$ Relation with binaural unmasking}

548 The present algorithm was conceived as a pre-processing tool to improve the SNR. However,

549 we incidentally found that it can coarsely simulate binaural intelligibility differences (BILDs).

550 The BILD is defined as the SRT improvement that results from having dichotic rather than diotic

551 speech-in-noise stimuli (Levitt and Rabiner, 1967). In free-field listening, it may be defined as

552 the SRT improvement (decrease) for any target-masker spatial configuration relative to the

$553 \mathrm{~S}_{0} \mathrm{~N}_{0}$ condition, where stimuli are almost diotic (note that diotic stimuli is unlikely to occur in

554 natural listening situations due to small asymmetries between the ears). Because BILDs exceed

555 the benefit provided by the head shadow alone, i.e., the benefit from listening with the ear

556 that has the better acoustic SNR, (e.g. Bronkhorst and Plomp, 1998), the current view is that

557 BILDs also reflect some form of binaural interaction in the central auditory system to 'cancel'

558 the masker(s) (e.g., Durlach, 1963; Culling, 2007; Hauth and Brand, 2018).

559 Figure 12A shows experimental BILDs for a target at $0^{\circ}$ azimuth in competition with a single

560 SSN source as a function of masker azimuth (denoted as the $\mathrm{S}_{0} \mathrm{~N}_{\mathrm{X}}$, with $\mathrm{X}$ indicating the masker

561 azimuth). Figures 12B and 12C show BILDs for configurations that included a second SSN

562 source at $105^{\circ}$ azimuth $\left(\mathrm{S}_{0} \mathrm{~N}_{105} \mathrm{~N}_{\mathrm{x}}\right)$, and a third source at $255^{\circ}$ azimuth $\left(\mathrm{S}_{0} \mathrm{~N}_{105} \mathrm{~N}_{255} \mathrm{~N}_{\mathrm{x}}\right)$,

563 respectively. All data are from Peissig and Kollmeier (1997). For comparison, Figure 12D-F

564 show the SNR improvements relative to the $\mathrm{S}_{0} \mathrm{~N}_{0}$ condition produced by the present algorithm 
565 for corresponding listening conditions and for two sets of weights: $\alpha_{\text {diff } f}$ and $\beta_{\text {diff }}$, and $\alpha_{270 \pm 30}$

566 and $\beta_{90 \pm 30}$. The former were calculated using Eq. 5 by averaging KEMAR HRTFs over the

567 azimuth range $0^{\circ}-180^{\circ}$ for $\beta(f)$ and $180^{\circ}-360^{\circ}$ for $\alpha(f)$, while the latter were calculated using

568 Eq. (5) but averaging KEMAR HRTFs over a narrower azimuth range of $60^{\circ}-120^{\circ}$ for $\beta(f)$ and

$569240^{\circ}-300^{\circ}$ for $\alpha(f)$. Also shown are SNR improvements for unprocessed stimuli, i.e.,

570 improvements produced by the head-shadow alone. Plots show the SNR in the ear with the

571 largest SNR for any given masker azimuth, which is equivalent to assuming that listeners use

572 the ear with the better acoustic SNR to recognize the speech. As the figure shows, the

573 algorithm with weights $\alpha_{270 \pm 30}$ and $\beta_{90 \pm 30}$ produces SNR improvements that are larger but

574 roughly similar to the pattern of experimental BILDs. In addition, SNR improvements are much

575 larger for processed than for unprocessed stimuli.

576 Based on the similarity between the pattern of SNR improvements and experimental BILDs in

577 Fig. 12, it is tempting to speculate that the central auditory system could weight and subtract

578 the stimuli at the two ears, and that the proposed algorithm could inspire binaural intelligibility

579 models. The idea of binaural subtraction is not new and goes back to the equalization-

580 cancellation theory of Durlach (1963). He theorized that "the auditory system attempts to

581 eliminate the masking component by first transforming the stimuli presented to the two ears

582 so as to equalize the two masking components, and the subtracting". A main difference with

583 Durlach's theory is that the present algorithm involves fixed weighting of the stimuli presented

584 to the two ears rather than equalization of the masking components at the two ears.

585 Furthermore, the present findings suggest that the weighting might be based on the inter-

586 aural ratio of HRTFs averaged over an appropriate azimuth range. Further research is

587 necessary to investigate these ideas, and explore the potential of the present algorithm as a

588 model of binaural intelligiblity.

\section{Limitations}

591 The measured SRT improvements were generally smaller than predicted by $\Delta$ SNR. For

592 example, the measured mean SRT improvement in the $S_{270} \mathrm{~N}_{45}$ condition was $\sim 8.5 \mathrm{~dB}$, thus

593 smaller than one would expect from Fig. 5A ( $\sim 14 \mathrm{~dB})$ if listeners used the ear with the better

594 acoustic SNR to perform the SRT task. Likewise, the measured SRT improvement in the $\mathrm{S}_{270} \mathrm{~N}_{90}$

595 was also smaller than predicted by $\triangle S N R$ in Fig. $5 A$ ( 0.8 vs $\sim 4.0 \mathrm{~dB}$ ). The reason is uncertain.

596 Perhaps, listeners did not use the ear with the better SNR to perform the speech recognition 
597 task. Or, perhaps, the processed stimuli interfered binaurally in the auditory brain of the

598 listeners to prevent them from taking full advantage of the SNR provided by the algorithm in 599 the ear ipsilateral to the target (e.g., Jerger et al., 1993; Mussoi and Bentler, 2017). The latter 600 explanation is partially supported by the fact that the measured SRT improvements were 601 greater, and closer to $\triangle$ SNR values, in unilateral than in bilateral listening (compare Fig. 8B 602 with Fig. 8D). The latter explanation is, however, insufficient because the observed SRT 603 improvements in unilateral listening were still smaller than predicted by $\Delta S N R$. Whatever the 604 reason, the present findings show that it would be wrong to expect actual SRT improvements 605 to be as large as $\triangle$ SNR.

606 As explained in the Introduction, pre-processing algorithms like the one proposed here are 607 often intended to improve the SNR to the users of assistive listening devices. The present tests 608 were limited to listeners with normal-hearing listeners. Further research is required to 609 investigate the benefits provided by the present algorithm when used in combination with 610 hearing aids and/or cochlear implants.

\section{Final remarks}

612 Given that listeners can use better-ear glimpsing in cocktail party scenarios (Brungart and lyer, 2012), and that the proposed algorithm can improve the SNR in the ear ipsilateral to the target source, the algorithm can improve the intelligibility of speech in competition with one or more sound sources without the user's intervention, without making any assumption about the temporal or spectral characteristics of the target and masker sources, and without making assumptions about the specific location of the interfering sound sources. In broad terms, it functions as a binaural beamformer that attenuates (or cancels) the contralateral sound field (Fig. 2), and whose frequency-dependent directivity pattern depends on the weights $\alpha(f)$ and $\beta(f)$ (Fig. 11). It operates in the frequency domain and its implementation in a hearing device would require two microphones (one per ear), and a means of exchanging the stimulus spectrum across the ears. Overall, the present algorithm is conceptually simpler and arguably easier to implement than many SNR enhancement pre-processing approaches (reviewed by Hamacher et al., 2005; Baumgärtel et al. 2015a; see the Introduction), thus potentially useful for implementation in binaural hearing devices. enhance low-frequency ILDs without the need of estimations of auditory cues or distorting the incoming sound" (their p. 79). Their algorithm effectively operates as a binaural beamformer 
that attenuates the contralateral sound field (see their Fig. 1B). On the other hand, the equalization-cancellation model was conceived to explain binaural masking level differences. In contrast with those approaches, the present algorithm was conceived as a practical solution to the general problem of cancelling out as many maskers as possible in cocktail party listening scenarios without having to know a priori the spectra or the locations of the target or the maskers (see section entitled The Algorithm). Given the disparity in motivations, it is remarkable that the present algorithm operates as a binaural beamformer that enhances head-shadow ILDs, as the algorithm of Dieudonne and Francart (2018) does, and that when set with appropriate values for $\alpha$ and $\beta$ (Fig. 12), it qualitatively simulates binaural unmasking in the free field, as the equalization-cancellation model does.

\section{CONCLUSIONS}

1. The proposed algorithm (binaural weighted subtraction of the contralateral stimulus) attenuates contralateral sounds, which in free-field listening conditions improves the SNR in the ear ipsilateral to the target sound source.

2. The subtraction weights may be set as needed. However, setting them equal to the ratio of ipsilateral to contralateral HRTFs averaged over an appropriate azimuth range can improve the SNR by as much as $20 \mathrm{~dB}$, and in multiple listening scenarios.

3. Binaural weighted subtraction can improve speech reception thresholds in noise by up 8.5 $\mathrm{dB}$ in bilateral listening and $10 \mathrm{~dB}$ in unilateral listening.

4. Binaural weighted subtraction using HRTF-based weights qualitatively accounts for binaural unmasking when speech is presented in competition with multiple maskers and for multiple target-masker spatial arrangements.

5. Binaural HRTF-weighted subtraction is simple and effective, which makes it potentially suitable for implementation in binaural hearing devices.

\section{ACKNOLWEDGEMENTS}

We thank Milagros J. Fumero for help with data collection, and Miriam I. Marrufo-Pérez, Peter T. Johannesen and Thibaud Leclere for useful discussions. Work supported by the Spanish Ministry of Science and Innovation (grant PID2019-108985GB-I00) and by MED-EL GmbH (Innsbruck, Austria).

\section{DECLARATION OF CONFLICTING INTERESTS}

The Authors declare that there is no conflict of interest. 
660

661

662

663

664

665

666

667

668

669

670

671

673

674

675

676

677

678

679

680

681

682

683

684

685

686

\section{APPENDIX. THE ALGORITHM IN DETAIL}

In a listening scenario where the target source with spectrum, $S(f)$, is presented in competition with multiple $(n)$ maskers with spectra $M_{1}(f), M_{2}(f), \ldots, M_{n}(f)$, the stimulus spectrum in the left and right ears, $L(f)$ and $R(f)$, can be calculated as follows:

$$
\begin{aligned}
& L(f)=S(f) H_{S L}(f)+\sum_{i=1}^{n}\left[M_{i}(f) H_{M_{i} L}(f)\right] \\
& R(f)=S(f) H_{S R}(f)+\sum_{i=1}^{n}\left[M_{i}(f) H_{M_{i} R}(f)\right]
\end{aligned}
$$

where $f$ denotes frequency $(\mathrm{Hz})$, and $H_{S L}(f), H_{M_{i} L}(f)$ are the complex HRTFs for the target $(S)$ and the $i$-th masker $\left(M_{i}\right)$ for the left $(L)$ ear, and $H_{S R}(f)$ and $H_{M_{i} R}(f)$ are the corresponding HRTFs for the right $(R)$ ear.

Substituting Eqs. (A1a) and (A1b) into Eq. (1a) and Eq. (1b), we get:

$L^{\prime}(f)=S(f) H_{S L}(f)+\sum_{i=1}^{n}\left[M_{i}(f) H_{M_{i} L}(f)\right]-\beta(f)\left\{S(f) H_{S R}(f)+\sum_{i=1}^{n}\left[M_{i}(f) H_{M_{i} R}(f)\right]\right\}$ (A2a)

$R^{\prime}(f)=S(f) H_{S R}(f)+\sum_{i=1}^{n}\left[M_{i}(f) H_{M_{i} R}(f)\right]-\alpha(f)\left\{S(f) H_{S L}(f)+\sum_{i=1}^{n}\left[M_{i}(f) H_{M_{i} L}(f)\right]\right\}$ (A2b)

For convenience, Eqs. (A2a) and (A2b) may be re-written as follows:

$$
\begin{aligned}
& L^{\prime}(f)=S(f) H_{S L}(f) A_{S}(f)+\left[\sum_{i=1}^{n} M_{i}(f) H_{M_{i} L}(f)\right] A_{M}(f) \\
& R^{\prime}(f)=S(f) H_{S R}(f) B_{S}(f)+\left[\sum_{i=1}^{n} M_{i}(f) H_{M_{i} R}(f)\right] B_{M}(f)
\end{aligned}
$$

Where:

$$
\begin{aligned}
& A_{S}(f)=1-\beta(f) \frac{H_{S R}(f)}{H_{S L}(f)}, \quad \text { and } \quad A_{M}(f)=1-\beta(f) \frac{\sum_{i=1}^{n} M_{i}(f) H_{M_{i} R}(f)}{\sum_{i=1}^{n} M_{i}(f) H_{M_{i} L}(f)} \\
& B_{S}(f)=1-\alpha(f) \frac{H_{S L}(f)}{H_{S R}(f)}, \quad \text { and } \quad B_{M}(f)=1-\alpha(f) \frac{\sum_{i=1}^{n} M_{i}(f) H_{M_{i} L}(f)}{\sum_{i=1}^{n} M_{i}(f) H_{M_{i} R}(f)}
\end{aligned}
$$

The right-hand side of Eqs. (A3a) and (A3b) contain one term for the target, $S(f)$, plus one term for the maskers, $M_{i}(f)$. Perfect cancellation of the masker(s) at the two ears can be achieved by making the masker terms equal to zero. That is, perfect cancellation of the masker(s) in the left ear can be achieved by choosing $\beta(f)$ such that $A_{M}(f)=0$ for all frequencies. Similarly, perfect cancellation of the maskers in the right ear can be achieved by choosing $\alpha(f)$ such that $B_{M}(f)=0$ for all frequencies.

From Eq. (A4a), it can be easily shown that $A_{M}(f)=0$ if and only if: 


$$
\beta(f)=\frac{\sum_{i}^{n} M_{i}(f) H_{M_{i} L}(f)}{\sum_{i}^{n} M_{i}(f) H_{M_{i} R}(f)}
$$

688

689

690

691

692

693

694

695

696

697

698

699

700

701

702

703

704

705

706

707

708

709

710

711

Similarly, from Eq. (A4b), $B_{M}(f)=0$ if and only if:

$$
\alpha(f)=\frac{\sum_{i}^{n} M_{i}(f) H_{M_{i} R}(f)}{\sum_{i}^{n} M_{i}(f) H_{M_{i} L}(f)}
$$

Note that:

\section{REFERENCES}

Baltzell LS, Swaminathan J, Cho AY, Lavandier M, Best V. (2020). Binaural sensitivity and release from speech-on-speech masking in listeners with and without hearing loss. J Acoust Soc Am 147:1546.

Baumgärtel RM, Hu H, Krawczyk-Becker M, Marquardt D, Herzke T, Coleman G, Adiloğlu K, Bomke K, Plotz K, Gerkmann T, Doclo S, Kollmeier B, Hohmann V, Dietz M. (2015b). Comparing binaural pre-processing strategies II: Speech intelligibility of bilateral cochlear implant users. Trends Hear 19. doi: 10.1177/2331216515617917.

Baumgärtel RM, Krawczyk-Becker M, Marquardt D, Völker C, Hu H, Herzke T, Coleman G, Adiloğlu K, Ernst SM, Gerkmann T, Doclo S, Kollmeier B, Hohmann V, Dietz M. (2015a) Comparing binaural pre-processing strategies I: Instrumental evaluation. Trends Hear 19. doi: $10.1177 / 2331216515617916$.

Best V, Swaminathan J. (2019). Revisiting the detection of interaural time differences in listeners with hearing loss. J Acoust Soc Am 145:EL508-EL513.

Boll SF. (1979). Suppression of acoustic noise in speech using spectral subtraction. IEEE Trans. Acoust. Speech Signal Process. 27:113-120.

Bronkhorst AW, Plomp R. (1988). The effect of head-induced interaural time and level differences on speech intelligibility in noise. J Acoust Soc Am 83:1508-1516.

Bronkhorst AW, Plomp R. (1989). Binaural speech intelligibility in noise for hearing-impaired listeners. J Acoust Soc Am 86:1374-1383.

Brown CA. (2014). Binaural enhancement for bilateral cochlear implant users. Ear Hear 35:580584. 
Brown CA. (2018). Corrective binaural processing for bilateral cochlear implant patients. Plos ONE 13(1):e0187965. https://doi.org/10.1371/journal.pone.0187965

Brungart DS, Iyer N. (2012). Better-ear glimpsing efficiency with symmetrically-placed interfering talkers. J Acoust Soc Am 132:2545-2556.

Burkhard MD, Sachs RM. (1975). Anthropometric manikin for acoustic research. J Acoust Soc Am 58:214-222. doi:10.1121/1.380648

Cherry EC. (1953). Some experiments on the recognition of speech with one and with two ears. J Acoust Soc Am 25:975-979.

722

Cornelis B, Moonen M, Wouters J. (2012). Speech intelligibility improvements with hearing aids using bilateral and binaural adaptive multichannel Wiener filtering based noise reduction. J Acoust Soc Am 131:4743-4755.

Cox H, Zeskind RM, Owen MM. (1987). Robust adaptive beamforming. IEEE Trans Acoustics, Speech, Signal Processing 35:1365-1376.

Culling JF. (2007). Evidence specifically favoring the equalization-cancellation theory of binaural unmasking. J Acoust Soc Am 122:2803-2813.

Doclo S, Kellermann W, Makino S, Nordholm S. (2015). Multichannel signal enhancement algorithms for assisted listening devices: Exploiting spatial diversity using multiple microphones. IEEE Signal Process Mag 32:18-30.

Durlach NI. (1963). Equalization and cancellation theory of binaural masking-level differences. J Acoust Soc Am 35:1206-1218.

Festen JM, Plomp R. (1990). Effects of fluctuating noise and interfering speech on the speechreception threshold for impaired and normal hearing. J Acoust Soc Am 88:1725-1736.

Gardner WG, Martin KD. (1995). HRTF measurements of a KEMAR. J Acoust Soc Am 97:39073908.

Grange JA, Culling JF. (2016). The benefit of head orientation to speech intelligibility in noise. J Acoust Soc Am 139:703-712.

Grantham, D., Ashmead, D., Ricketts, T., Haynes, D., Labadie, R. (2008). Interaural time and level difference thresholds for acoustically presented signals in post-lingually deafened adults fitted with bilateral cochlear implants using CIS+ processing. Ear Hear 29:33-44. 
Hamacher V, Chalupper J, Eggers J, Fischer E, Kornagle U, Puder H, Rass U. (2005). Signal processing in high-end hearing aids: State of the art, challenges and future trends. EURASIP J Appl Sig Process 18:2915-2929.

Hauth CF, Brand T. (2018). Modeling sluggishness in binaural unmasking of speech for maskers with time-varying interaural phase differences. Trends Hear doi: $\underline{10.1177 / 2331216517753547}$

Hochmuth S, Brand T, Zokoll MA, Castro FZ, Wardenga N, Kollmeier B. (2012). A Spanish matrix sentence test for assessing speech reception thresholds in noise. Int J Audiol 51:536-544. doi:10.3109/14992027.2012.670731

Holube I, Fredelake S, Vlaming M, Kollmeier B. (2010). Development and analysis of an international speech test signal (ISTS). Int J Audiol 49:891-903.

Huarte A. (2008). The Castilian Spanish hearing in noise test. Int J Audiol 47:369-370. doi:10.1080/14992020801908269

Jerger J, Silman S, Lew HL, Chmiel R. (1993). Case studies in binaural interference: converging evidence from behavioral and electrophysiologic measures. J Am Acad Audiol 4:122-131.

Kokkinakis K, Pirhosseinloo S. (2017). An algorithm for enhancement of naturally occurring level differences in bilateral cochlear implant users. 2017 Conference on Implantable Auditory Prostheses, 16-21 July 2017, Lake Tahoe, California.

Levitt H, Rabiner LR. (1967). Binaural release from masking for speech and gain in intelligibility. J Acoust Soc Am 42:602-608.

Levitt H. (1971). Transformed up-down methods in psychoacoustics. J Acoust Soc Am 49:467477.

Liu C, Wheeler BC, O'Brien WD Jr, Lansing CR, Bilger RC, Jones DL, Feng AS. (2001). A twomicrophone dual delay-line approach for extraction of a speech sound in the presence of multiple interferers. J Acoust Soc Am 110:3218-3231.

Lopez-Poveda EA, Eustaquio-Martín A, Fumero MJ, Gorospe JM, Polo López R, Gutiérrez Revilla MA, Schatzer R, Nopp P, Stohl JS. (2020). Speech-in-noise recognition with more realistic implementations of a binaural cochlear-implant sound coding strategy inspired by the medial olivocochlear reflex. Ear Hear 41:1492-1510. 
Lopez-Poveda EA, Eustaquio-Martín A, Fumero MJ, Stohl JS, Schatzer R, Nopp P, Wolford RD, Gorospe JM, Polo R, Gutiérrez Revilla MA, Wilson BS. (2019). Lateralization of virtual sound sources with a binaural cochlear-implant sound coding strategy inspired by the medial olivocochlear reflex. Hear Res 379:103-116.

Lopez-Poveda EA, Eustaquio-Martín A. (2018). Objective speech transmission improvements with a binaural cochlear implant sound-coding strategy inspired by the contralateral medial olivocochlear reflex. J Acoust Soc Am 143:2217-2231.

Lopez-Poveda EA, Eustaquio-Martin, A, Stohl JS, Wolford RD, Schatzer R, Gorospe JM, Santa Cruz Ruiz S, Benito F, Wilson BS (2017). Intelligibility in speech maskers with a binaural cochlear implant sound coding strategy inspired by the contralateral medial olivocochlear reflex. Hear Res 348:134-137.

Lopez-Poveda EA, Eustaquio-Martin, A, Stohl JS, Wolford RD, Schatzer R, Wilson BS. (2016a). A binaural cochlear implant sound coding strategy inspired by the contralateral medial olivocochlear reflex. Ear Hear 37:e138-e148.

Lopez-Poveda EA, Eustaquio-Martin, A, Stohl JS, Wolford RD, Schatzer R, Wilson BS. (2016b). Roles of the contralateral efferent reflex in hearing demonstrated with cochlear implants. Adv Exp Med Biol 894:105-114.

Luts H, Eneman K, Wouters J, Schulte M, Vormann M, Buechler M, Dillier N, Houben R, Dreschler WA, Froehlich M, Puder H, Grimm G, Hohmann V, Leijon A, Lombard A, Mauler D, Spriet A. (2010). Multicenter evaluation of signal enhancement algorithms for hearing aids. J Acoust Soc Am 127:1491-1505.

Moore BCJ, Kolarik A, Stone MA, Lee Y-W (2016). Evaluation of a method for enhancing interaural level differences at low frequencies. J Acoust Soc Am 140:2817-2828.

Moore BCJ. (2007). Binaural sharing of audio signals: Prospective benefits and limitations. Hear J 60:46-48.

Mussoi BSS, Bentler RA. (2017). Binaural interference and the effects of age and hearing loss. J Am Acad Audiol 28:5-13.

Peissig J, Kollmeier B. (1997). Directivity of binaural noise reduction in spatial multiple noisesource arrangements for normal and impaired listeners. J Acoust Soc Am 101:1660-1670. 
801 Peters RW, Moore BCJ, Baer T. (1998). Speech reception thresholds in noise with and without

802 spectral and temporal dips for hearing-impaired and normally hearing people. J Acoust Soc

803 Am 103:577-587.

804 Taal CH, Hendriks RC, Heusdens R, Jensen J. (2011). An algorithm for intelligibility prediction of

805 time-frequency weighted noisy speech. IEEE Trans Audio Speech Lang Process 19:2125-

8062136.

807 Wiggins IM, Seeber BU. (2013). Linking dynamic-range compression across the ears can

808 improve speech intelligibility in spatially separated noise. J Acoust Soc Am 133:1004-1016.

809 


\section{FIGURE CAPTIONS}

811 Figure 1. The magnitude (top) and phase (bottom) spectra of $\alpha_{\text {diff }}$ and $\beta_{\text {diff }}$ (Eq. 5) calculated using the HRTFs for a KEMAR (Gardner and Martin, 1995). Note that $\alpha_{\text {diff }}=\beta_{\text {diff }}$ (Eq. 7) and that the phase is unwrapped.

814 Figure 2. Directivity patterns for the left ear with and without processing using $\beta_{\text {diff }}$. Patterns

815 are shown for white noise (left panels) and speech-shaped noise (right panels). The top and

816 bottom panels show patterns for the low- $(0-1500 \mathrm{~Hz})$ and high-frequency (>1500 Hz) content

817 of each noise stimulus, respectively. The directivity pattern for the right ear would be

818 symmetrical about the midline.

Figure 3. Example spectrograms for unprocessed (middle row) and processed (bottom row) stimuli in the left and right ears, respectively. The target was the Spanish word "sastre" at $270^{\circ}$ azimuth in competition with a single source of speech-shaped noise at $45^{\circ}$. The SNR before HRTF filtering was $-10 \mathrm{~dB}$ SNR. For reference, the top panels illustrate the spectrograms for the HRTF-filtered speech alone.

824 Figure 4. As Fig. 3, but for white noise rather speech-shaped noise.

825 Figure 5. SNR improvement $(\triangle S N R)$ in the left and right ears provided by the algorithm with

$826 \beta_{\text {diff }}$ and $\alpha_{\text {diff }}$ for listening scenarios with the target and one masker source at azimuths from

$8270^{\circ}$ to $360^{\circ}$ every $15^{\circ}$. The color bar indicates the SNR improvement in decibels. Positive and

828 negative values indicate higher and lower SNR for processed than for unprocessed stimuli,

829 respectively. Dashed-dotted lines along the diagonals depict conditions with collocated target

830 and masker sources. The dotted lines and numbers indicate the quadrants referred to in the

831 main text.

Figure 6. STOI improvement ( $\triangle \mathrm{STOI}$ ) for the left and right ears provided by the algorithm for various azimuthal locations of the target and masker sources in the horizontal plane. The SNR of the unprocessed stimuli before HRTF filtering was $0 \mathrm{~dB}$. The color bar indicates the STOI improvement. Positive and negative values indicate higher and lower STOI for processed than for unprocessed stimuli, respectively. Circles depict the three target-masker spatial configurations $\left(\mathrm{S}_{0} \mathrm{~N}_{0}, \mathrm{~S}_{270} \mathrm{~N}_{45}\right.$, and $\left.\mathrm{S}_{270} \mathrm{~N}_{90}\right)$ used in the experimental evaluation of the algorithm.

838 Figure 7. Improvement in STOI (A, $\triangle$ STOI) and SNR (B, $\triangle$ SNR) in the left ear for 10 simulated 839 listening scenarios with different number of maskers, and azimuth and masker locations. For 840 each scenario, results are shown for three masker types (IFFM, SSN, WN). For scenarios \#1 to $841 \# 7$, results are shown for $\alpha_{\text {diff }}$ and $\beta_{\text {diff }}$ calculated using individualized and non-individualized 
842 HRTFs. Bars terminated with an arrow indicate that the actual bar extends beyond the corresponding $\mathrm{x}$-axis range. Note that in some conditions, $\triangle \mathrm{STOI}$ and/or $\triangle \mathrm{SNR}$ are equal to zero and hence the bar is not visible. See the main text for details.

Figure 8. The effect of processing on speech-in-noise recognition for selected target-masker processor. In all panels, circles illustrate individual data, bars illustrate group mean scores $(\mathrm{N}=5)$, and error bars illustrate plus and minus one standard deviation. Unilateral listening involved listening with the left ear (i.e., the ear ipsilateral to the target source in the $S_{270} N_{45}$, and $\mathrm{S}_{270} \mathrm{~N}_{90}$ configurations). ${ }^{* *} p \leq 0.01,{ }^{* *} p \leq 0.001$.

Figure 9. Top. The level at the left and right ears for unprocessed and processed stimuli. The stimulus level before HRTF filtering was $-20 \mathrm{~dB}$ FS. Bottom. Inter-aural level difference (ILD) for unprocessed and processed stimuli. Each column is for a different noise bandwidth, as indicated at the top: white $(0-22050 \mathrm{~Hz})$, broadband $(125-6000 \mathrm{~Hz})$, and lowband (125-1000 $\mathrm{Hz})$.

Figure 10. The effect of processing on the localization of lowband (LBN) and broadband (BBN) noise bursts in a virtual horizontal plane. Open and filled bars illustrate group mean angle error scores for unprocessed and processed stimuli, respectively. Error bars illustrate plus and minus one standard deviation of the group mean. Circles illustrate individual scores.

Figure 11. SNR improvement produced by the algorithm in left ear for a target at $0^{\circ}$ azimuth in competition with a SSN source at azimuths from $0^{\circ}$ to $360^{\circ}$ (in steps of $15^{\circ}$ ). Each trace illustrates the improvement for different values of $\beta(f)$ calculated using Eq. (5a) but averaging KEMAR HRTFs over different azimuth ranges, as indicated by the inset. The improvement is relative to HRTF-filtered but otherwise unprocessed stimuli. See the main text for details.

867 Figure 12. Comparison of the SNR provided by the present algorithm in the ear with the largest 868 SNR (right panels) with experimental SRTs (data from Peissig and Kollmeier, 1997) (left panels) relative to a spatial configuration with the target and masker collocated at $0^{\circ}$ azimuth. Lower values indicate better performance. Note the different ordinate scales in the left and right panels. The top, middle, and bottom rows show results for a target at $0^{\circ}$ azimuth in competition with one, two, and three different SSN sources, respectively. The abscissa 
bioRxiv preprint doi: https://doi.org/10.1101/2021.01.22.427757; this version posted January 22, 2021. The copyright holder for this preprint (which was not certified by peer review) is the author/funder, who has granted bioRxiv a license to display the preprint in perpetuity. It is made available under aCC-BY 4.0 International license.

874 illustrate the results when the second SSN source, and third SSN source were located at $105^{\circ}$

875 and $255^{\circ}$ azimuth, respectively. See the main text for details.

876 

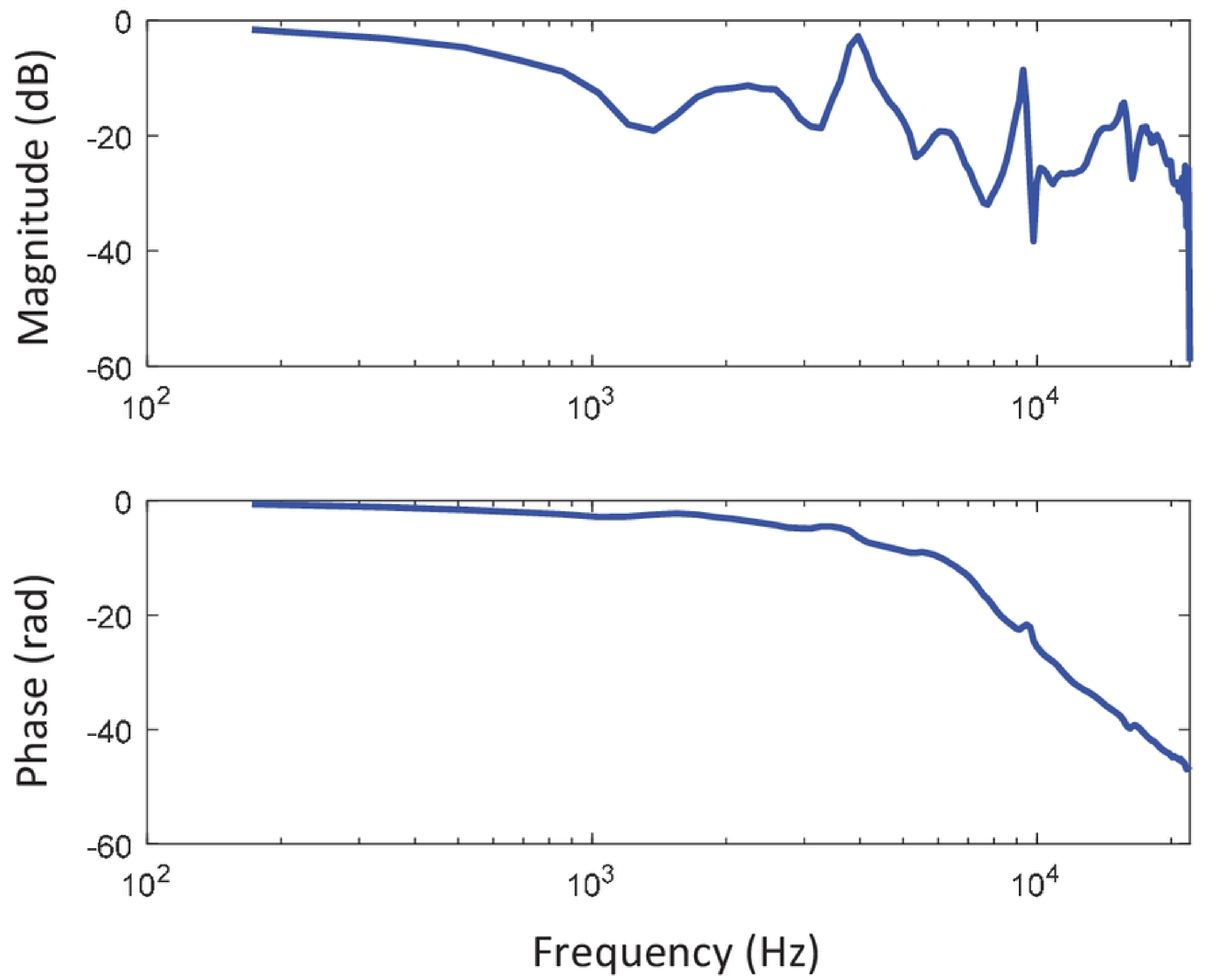

Figure 1 


\section{Target word}

Right ear

dB FS
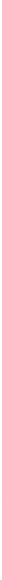

Unprocessed (target + white noise)
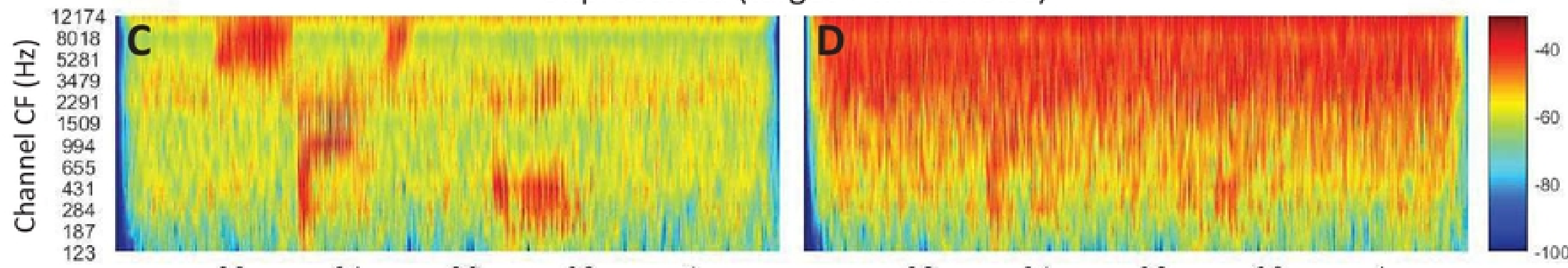

0.2

0.4

0.6

1

0.2

0.4

0.6

0.8

1

Processed (target + white noise)

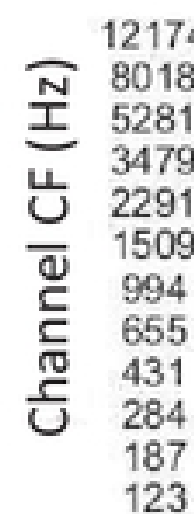

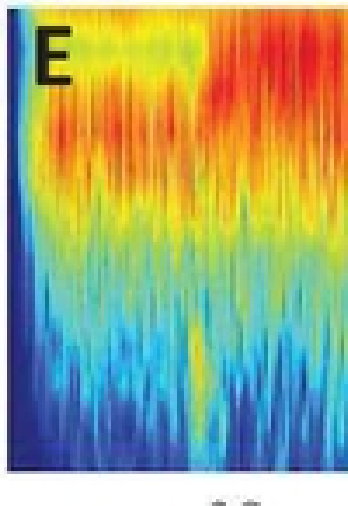

0.2

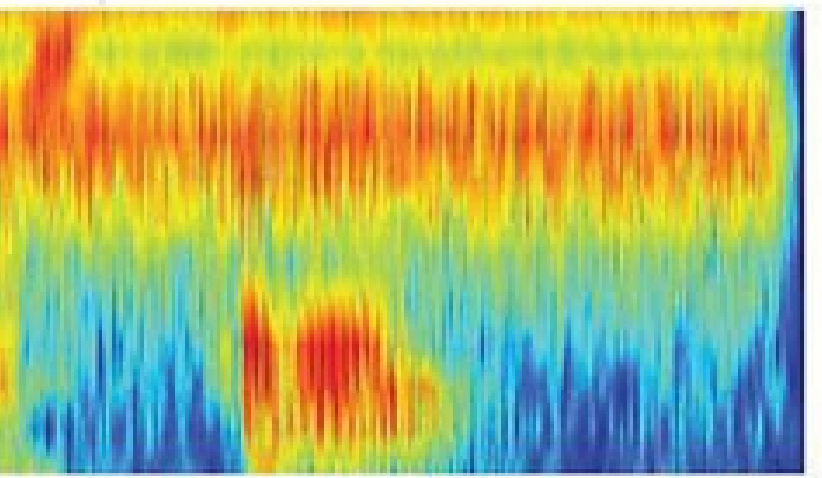

0.6

Time (s)

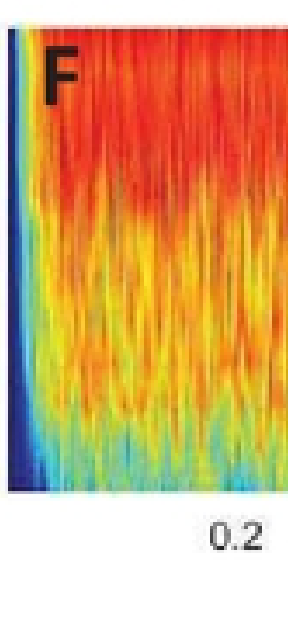

Figure 4 


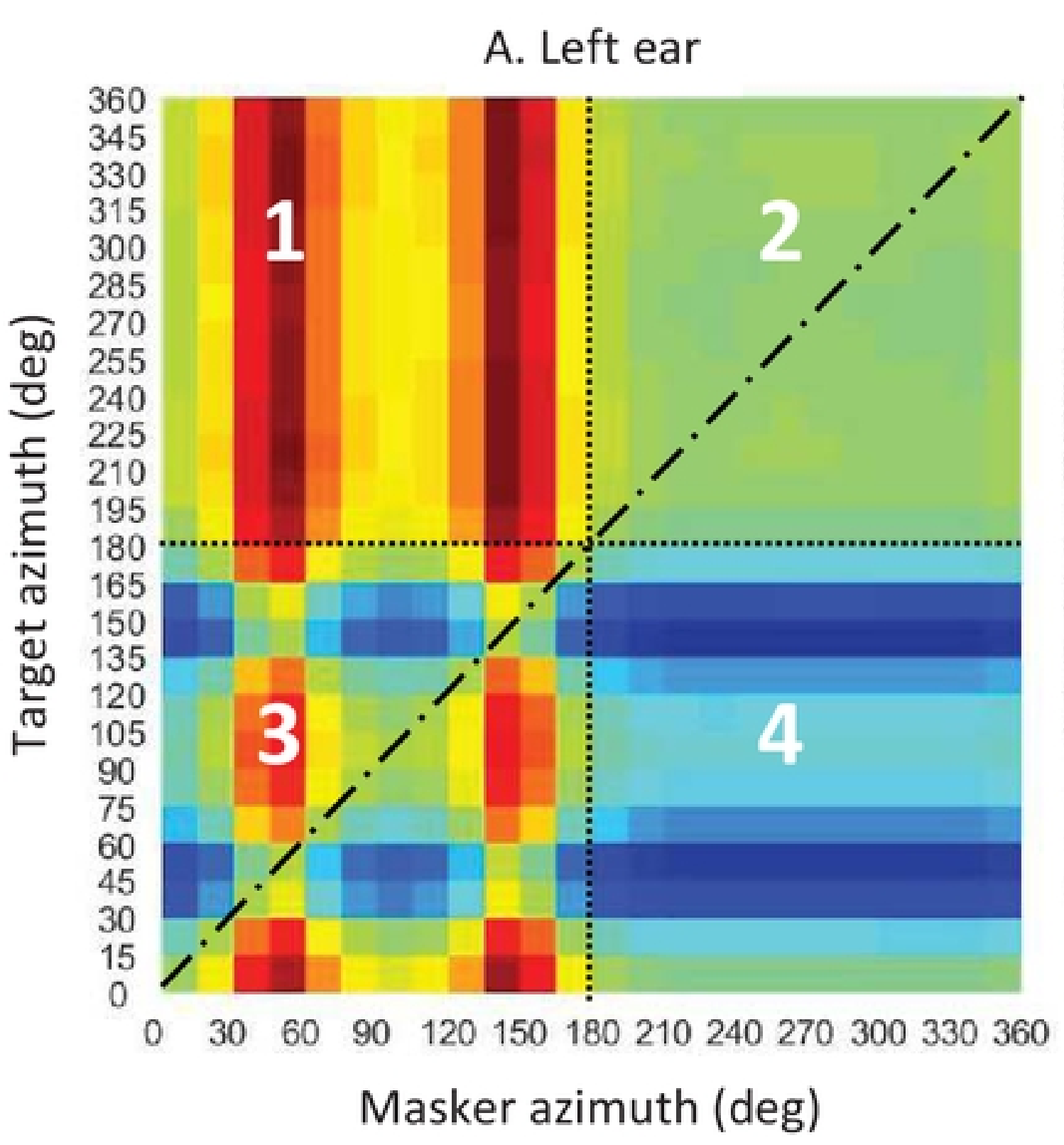

$\Delta \mathrm{SNR}$

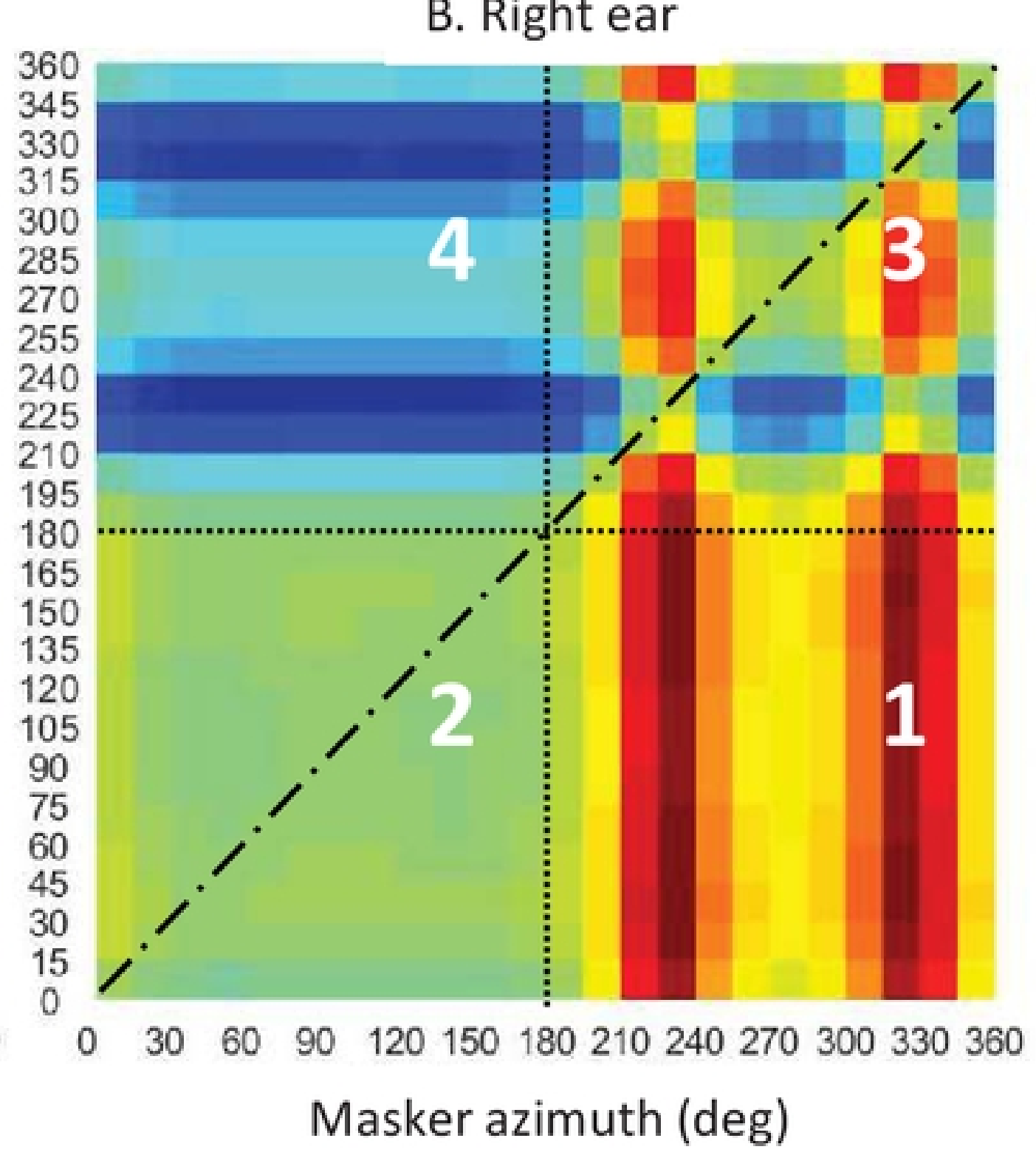

(dB)

Figure 5 
A. Left ear

B. Right ear

$\Delta$ STOI

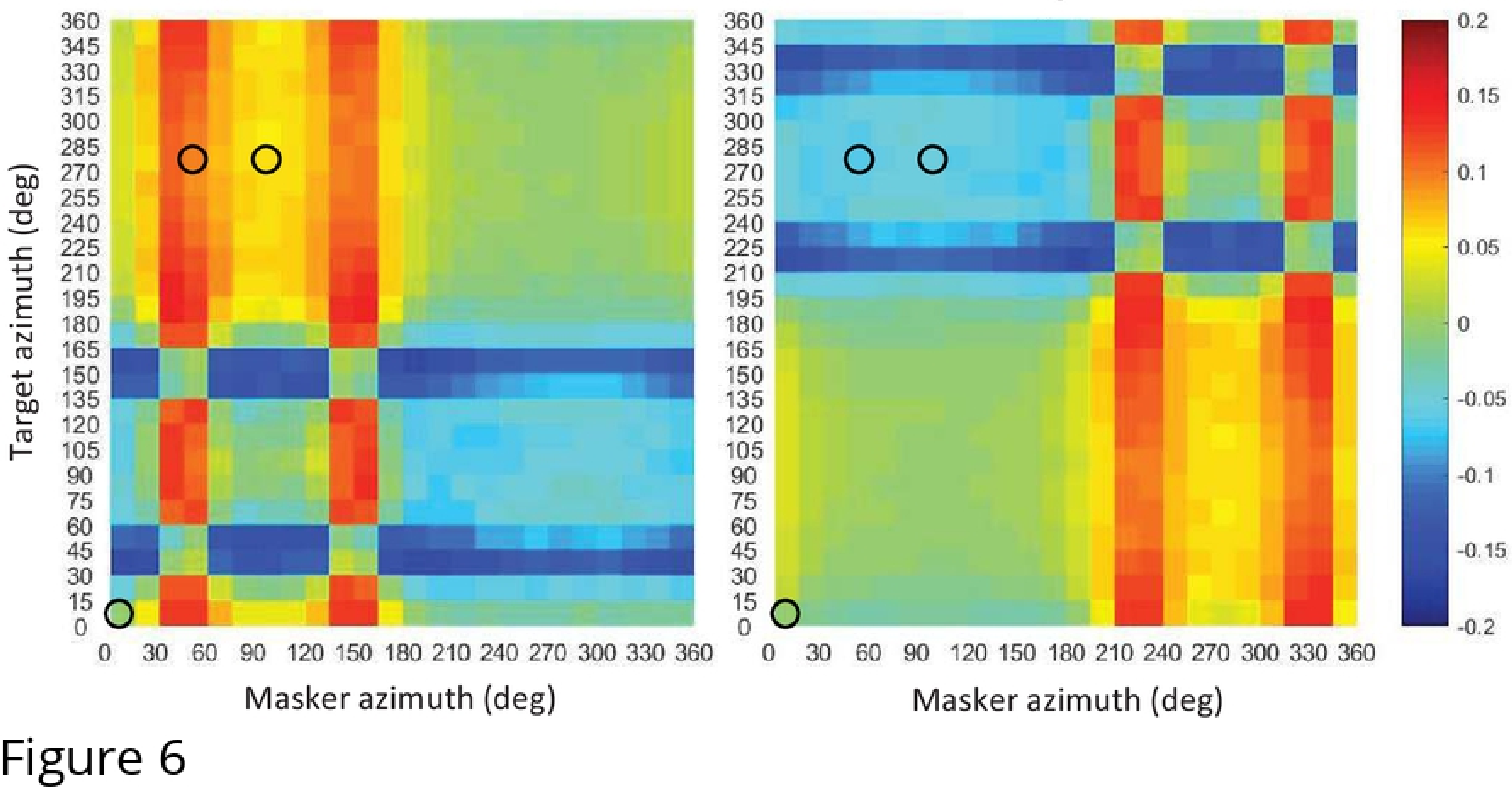


Peissig and Kollmeier (1996)

Present algorithm

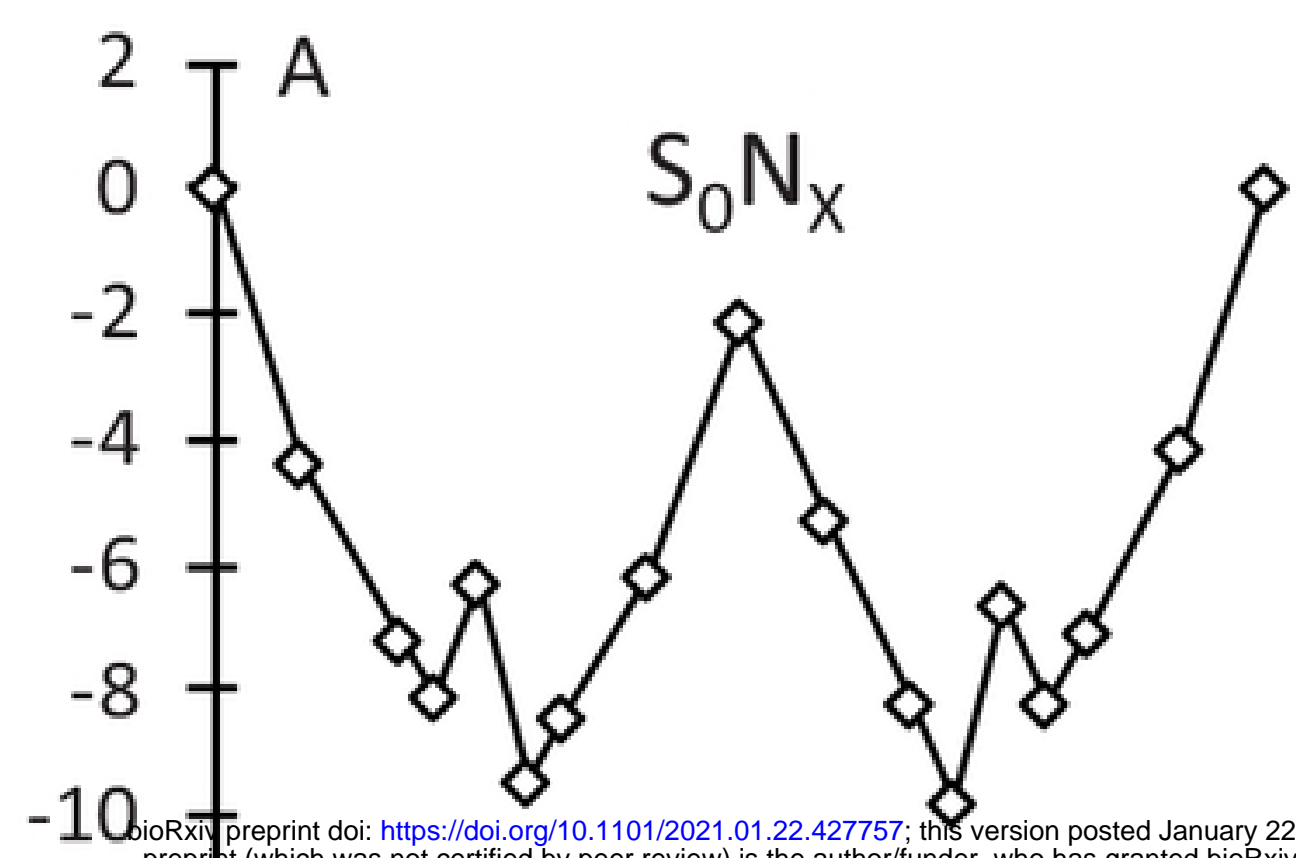

prepript (which was not certified by peer review) is the author/funder, who has granted bioRxiv a
perpetuity. It is made available under CCC-BY 4.0 International lice

$\begin{array}{lllllll}0 & 60 & 120 & 180 & 240 & 300 & 360\end{array}$
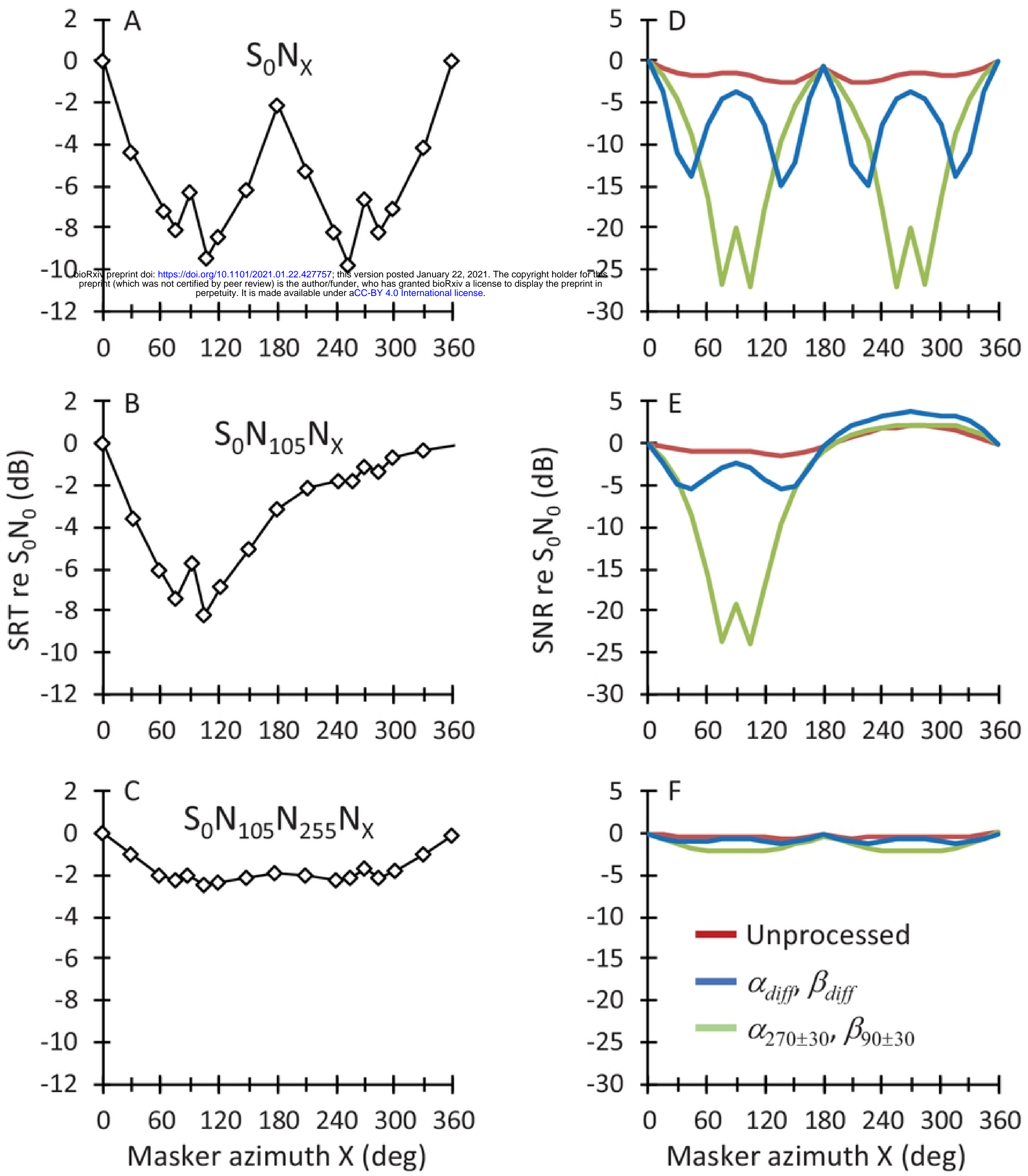

Figure 12

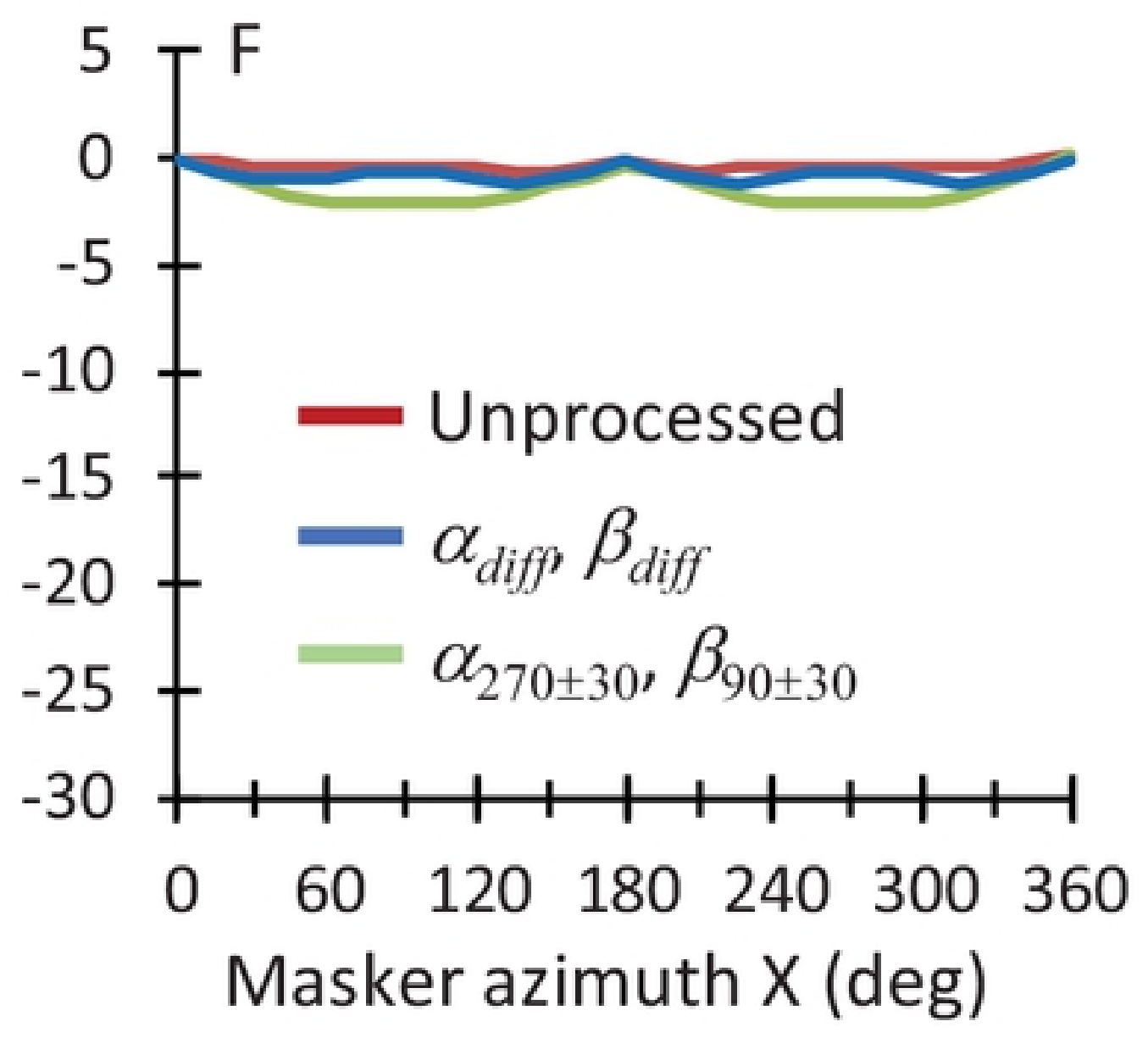

